\title{
Efficient mixing in stratified flows: Experimental study of a Rayleigh-Taylor unstable interface within an otherwise stable stratification
}

\author{
MEGAN S. DAVIES WYKES † AND STUART B. DALZIEL \\ Department of Applied Mathematics and Theoretical Physics, University of Cambridge, \\ Wilberforce Road, Cambridge CB3 0WA, UK
}

(Received ?; revised ?; accepted ?. - To be entered by editorial office)

Boussinesq salt-water laboratory experiments of Rayleigh-Taylor instability can achieve mixing efficiencies greater than 0.75 when the unstable interface is confined between two stable stratifications. This is much greater than that found when Rayleigh-Taylor instability occurs between two homogeneous layers when the mixing efficiency has been found to approach 0.5. Here, the mixing efficiency is defined as the ratio of energy used in mixing compared to the energy available for mixing. If the initial and final states are quiescent then the mixing efficiency can be calculated from experiments by comparison of the corresponding density profiles. Varying the functional form of the confining stratifications has a strong effect on the mixing efficiency. We derive a buoyancy-diffusion model for the rate of growth of the turbulent mixing region, $\dot{h}=2 \sqrt{\alpha A g h}$ (where $A=A(h)$ is the Atwood number across the mixing region when it extends a height $h, g$ is acceleration due to gravity and $\alpha$ is a constant). This model shows good agreement with experiments when the value of the constant $\alpha$ is set to 0.07 , the value found in experiments of Rayleigh-Taylor instability between two homogeneous layers (where the height of the turbulent mixing region increases as $h=\alpha A g t^{2}$, an expression which is equivalent to that derived for $\dot{h}$ ).

Key words: buoyancy-driven instability, turbulent mixing, mixing efficiency, RayleighTaylor instability

\section{Introduction}

Turbulent mixing in stratified flows is often characterised by a mixing efficiency. This mixing efficiency compares the energy used in irreversible mixing, increasing the minimum gravitational potential energy that can be stored in the density field, to the total change in mechanical energy during the mixing process. It can be defined either as an integral quantity, calculated between quiescent initial and final conditions or as a ratio of the energy flux to mixing and the power into the system. These two definitions can give different values if the system is not in steady state. Mixing efficiency is particularly significant in oceanography as mixing is required to maintain the overall stratification in a steady state ocean (Munk \& Wunsch 1998). The total amount of mixing in the oceans is equal to the product of the power input to the ocean and the average mixing

$\dagger$ Email address for correspondence: megan.davieswykes@cantab.net 


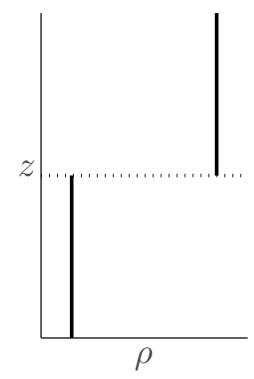

(a)

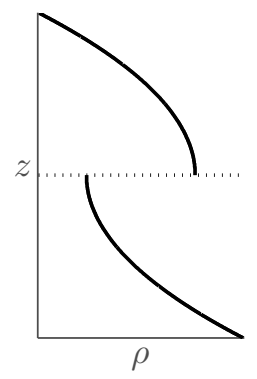

(b)

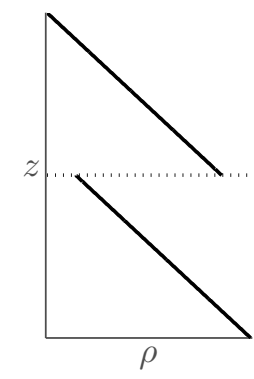

(c)

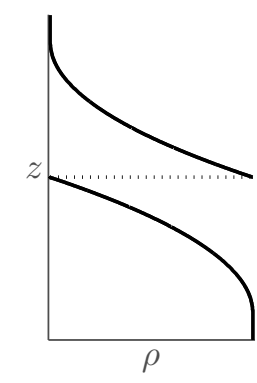

(d)

Figure 1: Sketch of (a) the classical case of Rayleigh-Taylor instability (a layer of dense fluid above a layer of light fluid) and (b-d) the initial density stratifications used in experiments. The two quadratic profiles $(\mathrm{b}, \mathrm{d})$ differ by having a density gradient that either increases $\left((\mathrm{b}) ; \frac{\mathrm{d}^{2} \rho}{\mathrm{d}|z|^{2}}>0\right)$ or decreases $\left((\mathrm{d}) ; \frac{\mathrm{d}^{2} \rho}{\mathrm{d}|z|^{2}}<0\right)$ away from the unstable interface.

efficiency (Wunsch \& Ferrari 2004). In this article we seek to gain a better understanding of turbulent mixing in general and, more specifically, of the limits of mixing efficiency.

For shear instability between two otherwise stably-stratified layers, a value for the mixing efficiency that is commonly quoted is $\sim 0.2$ (Peltier \& Caulfield 2003) and mixing efficiency is sometimes taken to be equal to this value in general. More recent laboratory experiments of exchange flows suggest that, for sufficiently high Reynolds number, the mixing efficiency of shear instability is closer to 0.11 (Prastowo et al. 2008). In comparison, the mixing efficiency of the Rayleigh-Taylor instability in laboratory experiments approaches $\frac{1}{2}$ (when the two homogeneous layers are of equal depth but different densities), though it can be shown that this value is the maximum possible for this configuration (Lawrie \& Dalziel 2011a). As Rayleigh-Taylor instability has a relatively high mixing efficiency, it appears to be a good candidate for investigating the upper limits of mixing efficiency.

Although the primary motivation of this article is to gain a greater understanding of the limits of mixing efficiency, additional motivations are in the area of oceanography. Examples of gravitationally unstable buoyancy driven mixing in the ocean include river in-flows, hydrothermal plumes and radiative cooling of the ocean surface and these flows must be characterised in ocean models. A more specific example is the breaking of internal waves, a flow which could potentially look similar to the density profiles investigated (figure 1).

Rayleigh-Taylor (RT) instability occurs when the pressure gradient opposes the density gradient, $\nabla p \cdot \nabla \rho<0$ (Rayleigh 1900; Taylor 1950). The classical case of this instability occurs when a layer of fluid with density $\rho_{u}$ is resting on a layer of fluid of density $\rho_{l}$, where $\rho_{u}>\rho_{l}$ and the whole system is in a gravitational field $g$. A sketch of this classical case can be seen in figure 1a. Perturbations in the interface between the two layers will grow. In general, for miscible fluids the two layers will mix together if the Reynolds number $\left(R e=\frac{u l}{\nu}\right.$, where $u$ and $l$ are flow velocity and length scales respectively and $\nu$ is the kinematic viscosity) becomes sufficiently high.

For sufficiently high Reynolds number, the governing parameter in Rayleigh-Taylor 
instability is the non-dimensional density difference or Atwood number,

$$
A_{0}=\frac{\rho_{u}-\rho_{l}}{\rho_{u}+\rho_{l}},
$$

where $\rho_{u}$ and $\rho_{l}$ are the densities of the fluid just above and below the Rayleigh-Taylor unstable interface. The Atwood number scales the effect of the destabilising acceleration and is related to the 'reduced gravity' by $g^{\prime}=2 A_{0} g$. We can also relate the Reynolds number to the Atwood number as, for a given experimental set-up, increasing $A_{0}$ will increase the maximum velocity in the flow, thereby increasing the energy contained in the large scales (the precise relation will be discussed in $\S 5$ ).

Due to the significance of RT instability in astrophysics (Cabot \& Cook 2006) and inertial confinement fusion (Petrasso 1994), there is a large body of literature, comprising experimental, numerical and theoretical studies. The reviews of Sharp (1984), Dimonte et al. (2004) and Andrews \& Dalziel (2010) examine previous work, which has mainly focused on the growth of the turbulent mixing region that develops between the two homogeneous layers of the classical instability.

When the instability is between two homogeneous layers, dimensional analysis shows that when the flow enters a high Reynolds number, self-similar regime, the penetration of the light fluid into the dense fluid (also known as the height of the mixing region, $h$ ) will develop as $h \sim f\left(A_{0}\right) g t^{2}$ (see Youngs 1984). If there is only a small density difference between the layers $\left(A_{0} \ll 1\right)$, the instability is symmetric: the light fluid rises into the dense fluid at the same rate as the dense fluid falls into the light fluid. There is a wealth of experimental and computational evidence to show that for $A_{0} \ll 1, f\left(A_{0}\right)=A_{0}$, making $h=\alpha A_{0} g t^{2}$, where $\alpha$ is a constant. This relationship can be derived in a variety of ways: through a buoyancy-drag model (Youngs 1984); an energy or force balance (Inogamov et al. 2001; Jacobs \& Dalziel 2005); through a bubble competition and merger model (Alon et al. 1995); or directly from the Navier-Stokes equations with an assumption that the density profile evolves self-similarly (Ristorcelli \& Clark 2004).

Although there have been many experimental studies (see Read (1984); Dalziel (1993); Linden et al. (1994); Snider \& Andrews (1994); Dalziel et al. (1999); Ramaprabhu \& Andrews (2004); Mueschke et al. (2009); Boffetta et al. (2010); Lister et al. (2011), and Tsiklashvili et al. (2012), among others), few examine anything other than the classical case of two layers of constant density. Exceptions include Jacobs \& Dalziel (2005), who examined the instability when both a stable and an unstable density interface were present in the system. In that study, evidence was found of mixing of the stable interface by turbulence generated in the RT unstable region.

Lawrie \& Dalziel (2011a) presented a series of experiments where a RT unstable interface was confined between two stable linear density profiles (a sketch of this configuration can be seen in figure 1c). They found that the mixing efficiency for this configuration was higher than that of the simple two-layer case, but experimental limitations prevented the determination of a precise value. In that paper they also derived a model for $h$ in this stratified case using an energy balance that required assuming a functional form for the density profile within the mixing region. A second paper by the same researchers examined RT instability, still confined by a stable linear density stratification, but now in a high aspect ratio tank $\left(\frac{H}{W}=40\right.$ where $H$ is the height and $W$ is the width of the tank) (Lawrie \& Dalziel 2011b). In this second paper, a hierarchy of models was presented: a zero-dimensional self-similar turbulent diffusion model for $h(t)$, a numerically integrated one-dimensional turbulent diffusion model for $\rho(z, t)$, and an implicit large eddy simulation (ILES) approach. ILES uses no explicit sub-gridscale model for dissipation, instead relying on numerical dissipation as a proxy for physical viscosity (Grinstein et al. 2007). 
The turbulent diffusion model in both the zero- and one-dimensional cases was derived using a balance between buoyancy and inertia. They found that $h$ was well described by a one-dimensional turbulent diffusion model.

In this paper we extend the work of Lawrie \& Dalziel (2011a), investigating the mixing efficiency of stratification confined Rayleigh-Taylor instability (RTI) for a variety of initial density profiles. These profiles are shown in figure $1(\mathrm{~b}-\mathrm{d})$. In $\S 2$ of this paper we describe what we mean by mixing in this context, define a mixing efficiency, and examine some possible final density profiles. Next, we describe the experimental procedures and density profiles used in $\S 3$. Our experimental results and analysis of the mixing will be presented in $\S 4$, where we describe how the initial density profile and Atwood number for an experiment affect mixing efficiency. We find that the final density profile in experiments (and therefore the mixing efficiency) can be predicted assuming that as the system nears equilibrium, the turbulent mixing region that developed on the unstable interface is well mixed (and thus nearly uniform in density), and the instability halts when the density profile is overall stable. In $\S 5$, we adapt the zero-dimensional, self-similar turbulent diffusion model employed by Lawrie \& Dalziel (2011b) to the standard low-aspect ratio geometry used in the present study and show that this model compares well with experiments. Finally, conclusions are presented in $\S 6$.

\section{Energetics and mixing}

In a closed system, we consider mixing to have occurred when the probability density function (pdf) of density has changed due to molecular diffusion. The mixing of two fluids of different densities is a two step process comprising stirring and diffusion. Diffusion is promoted by stirring, which stretches density surfaces and increases the density gradients across them. Rayleigh-Taylor instability is particularly effective at mixing, as there is a co-location of vorticity deposition by the baroclinic instability (which stretches fluid interfaces) and the density gradients that control diffusion (Lawrie \& Dalziel 2011a; Linden et al. 1994).

In the following analysis we will assume a linear equation of state for salt-water, where overall changes in internal energy are negligible compared to changes in the gravitational potential energy and kinetic energy. Under some oceanic conditions, the full non-linear equation of state for density, which is a function of temperature, pressure and salinity, $\rho=\rho(T, p, S)$, must be used (Tailleux 2009). However, in the laboratory, temperature changes of the environment are small (generally $T=19 \pm 1^{\circ} \mathrm{C}$ and the temperature change of the laboratory during an experiment is $\Delta T \ll 1^{\circ} \mathrm{C}$ ). Low Atwood numbers mean that the heat of dilution, released when the two fluids are mixed, is also small. These small temperature changes result in a negligible change in density and in the thermal expansion and haline contraction coefficients. The $O(1 \mathrm{~m})$ length-scales that occur in the laboratory mean that pressures do not vary significantly from atmospheric and water can be considered incompressible.

We ignore laminar diffusion associated with flat, horizontal interfaces as the time-scale for these processes is large, $\tau_{L} \sim \frac{H^{2}}{\kappa_{m}}$ (where $H$ is the height of the tank and $\kappa_{m}$ is the molecular diffusion coefficient) compared to the time-scale for the flux associated with RTI, $\tau_{T} \sim \sqrt{\frac{H}{A_{0} g}}$ (where $g$ is gravitational acceleration). We will assume a closed system where there are no transfers (advective or diffusive) of heat or mass across the boundaries of the system. Although the tank walls are not insulated, the temperature changes from ambient are small and so heat transfer to and from the environment is slow (mass transfer is small and will be discussed in $\S 3)$. 
In our experiments, only one-dimensional density measurements will be taken, and all horizontal density variations will be assumed to be zero $\left(\frac{\partial \rho}{\partial x}=\frac{\partial \rho}{\partial y}=0\right)$. This will be true so long as measurements of the density profile are taken when the experiment is quiescent. Other assumptions include that the fluids are miscible and that there is no surface tension or electrical potential energy between them, both valid for laboratory experiments using salt and fresh water.

\subsection{Mixing efficiency}

We wish to understand how the mixing process redistributes energy. Following Winters et al. (1995), we begin by partitioning gravitational potential energy in the flow,

$$
E_{p}=g \int_{V} z \rho \mathrm{d} V
$$

into that which is available to do work and background potential energy.

If every fluid parcel were allowed to rise and fall adiabatically and without mixing until in equilibrium, the system would have been rearranged into a state of minimum potential energy (Lorenz 1954). This can be considered to be our reference state (Tailleux 2013). The background potential energy $E_{b}$, is this minimum potential energy

$$
E_{b}=g \int_{V} z \hat{\rho} \mathrm{d} V
$$

where $\hat{\rho}$ is the rearranged density profile. Note that some authors use the equivalent expression of $E_{b}=g \int_{V} \hat{z} \rho \mathrm{d} V$, where $\hat{z}$ gives the vertical position of a parcel of fluid in the reference state (Winters et al. 1995). For many unstable profiles (two layers of constant density for example) there is not a unique $\hat{z}$, i.e. a parcel of fluid does not always have a unique position in the adiabatically rearranged state.

Allowing parcels of fluid of density $\rho(\mathbf{x}, t)$ to rise and fall until the system is in equilibrium effectively sorts the profile by density, which leaves us with the sorted density profile $\hat{\rho}(z, t)$ in which $\frac{\partial \hat{\rho}}{\partial z} \leqslant 0$. Note that the probability density functions of density for $\rho(\mathbf{x}, t)$ and $\hat{\rho}(z, t)$ are identical (i.e. the two density profiles contain the same amounts of each density). The background potential energy $E_{b}$ depends only on the pdf of density for the flow and not on the spatial distribution of density. In a closed system, only irreversible diabatic mixing can alter the pdf, reducing the variance of density and increase $E_{b}$. Energy locked-up by irreversible mixing of the density profile (directly associated with changes in the pdf of density) can be measured by calculating the change in $E_{b}$ between the initial and final states of an experiment (Winters et al. 1995).

The available potential energy of a flow is the change in the potential energy that would occur through the same adiabatic rearrangement we used to calculate $E_{b}$,

$$
E_{a}=E_{p}-E_{b}=g \int_{V} z(\rho-\hat{\rho}) \mathrm{d} V .
$$

The total energy that is available to do work includes any kinetic energy $E_{k}$ in the system and the available potential energy. This total available energy is

$$
E_{T}=E_{a}+E_{k}=\int_{V} \frac{1}{2} \rho|\mathbf{u}|^{2}+g z(\rho-\hat{\rho}) \mathrm{d} V .
$$

For a turbulent event that gives a change in the distribution of energy in the system, we can define a mixing efficiency which measures how much of the initially available energy in the system is used in (or lost by) irreversible mixing, as opposed to being dissipated 
by viscosity (neglecting any net changes in internal energy),

$$
\eta=\frac{\left|\Delta E_{b}\right|}{\left|\Delta\left(E_{a}+E_{k}\right)\right|}
$$

where $\Delta$ represents the change in the energy between the start and end states of the system. If we assume that the initial and final states of our experiment are quiescent $\left(\Delta E_{k}=0\right)$, this simplifies to

$$
\eta_{R T}=\frac{\left|\Delta E_{b}\right|}{\left|\Delta E_{a}\right|}
$$

We shall refer to this as the 'Rayleigh-Taylor mixing efficiency'.

Although Tailleux (2009) has pointed out that the route to background potential energy is via the internal energy of the fluid, it is instructive to consider a simplified view. In particular, diffusion acts to homogenise intermingled parcels of fluid of different densities (an arrangement containing available potential energy) to produce a more uniform density field with a higher background potential energy. Thus we can view diffusion as providing a flux of energy from $E_{a}$ to $E_{b}$. In our simplified view, during the evolution of an instability, available potential energy is converted into background potential energy though irreversible mixing. Conversion of available potential energy into kinetic energy also occurs and this assists the mixing of fluid parcels by stirring the flow. This kinetic energy is then either transferred back into available potential energy by raising the centre of mass of a fluid parcel or is dissipated by viscosity. Therefore energy is either used in mixing or is dissipated by viscosity.

For some flow systems, the initial configuration imposes limits on the mixing efficiency since once the density field is homogeneous, no further mixing is possible. For any purely odd monotonic statically unstable stratification, the mixing efficiency of RTI is limited to a maximum of $\frac{1}{2}$ (Lawrie \& Dalziel 2011a). In the simple two-layer case of RTI, when all the fluid has mixed until the system is homogeneous in density, it is possible that there is additional kinetic energy left in the system. However, as it is not possible for further mixing to occur, this additional kinetic energy must be dissipated by some other means, imposing a limit of $\frac{1}{2}$ on the mixing efficiency of the system.

As we are interested in the limits of mixing efficiency, we must examine a situation where the mixing is limited only by the energetics of the system and not by the selected initial configuration. This is the case when the interface is confined only by stable stratification, thereby removing the upper bound on the mixing that can be done and enabling the possibility of mixing efficiencies closer to the energetically imposed limit of 1 . We examine three cases, with varying curvature in the initial density profile $\frac{\partial^{2} \rho}{\partial|z|^{2}}$ (positive, negative or zero). The three cases are shown in figure 1(b-d). All the cases are anti-symmetric around $(z=0, \rho=\bar{\rho})$. The idealised equations for each density profile can be found in $\S 3$. First, however, we shall explore a range of feasible end-states for each stratification.

\subsection{Possible final profiles}

For a one-dimensional density profile in a closed system, the pdf is calculated by

$$
\operatorname{pdf}(\rho)=\left|\frac{1}{z_{+}-z_{-}} \frac{d z}{d \rho}\right|
$$

where the pdf is defined over the interval $z_{-}<z<z_{+}$. This is the fraction of height $d z$, occupied by each density $\rho$ (assuming $\rho(z)$ is a bijective function and $\frac{d z}{d \rho}<0$ ). In finding the pdf of a profile we lose information on the spatial distribution of density. For the 
initial RT unstable profiles $\rho(z)$ is not bijective, but each layer is separately. Therefore the initial pdfs of the upper and lower layers are calculated separately by (2.7), and the layer pdfs are added to find the total pdf of the whole system. Any mixing that occurs will change this total pdf, reducing the associated variance in the density field.

We will now examine some possible final profiles (see figure 2) and their corresponding pdfs (figure 3). Instability can be removed from a profile by some combination of mixing and sorting of fluid parcels. To get from some arbitrary unstable profile to equilibrium either the fluid must be mixed completely (removing unstable density gradients) or fluid parcels which are Rayleigh-Taylor unstable must pass each other (vertically) without completely mixing (resulting in a residual stratification in the final state). In this paper we will use the word 'sorting' to refer to both the physical process of fluid parcels moving vertically and adiabatically without complete mixing, and to the analytical process of sorting the elements of a vector or array by value as there is a strong analogy between the two.

The first situation we consider is where no mixing occurs. In this case all parcels of fluid rise and fall, without mixing, until the system is in equilibrium. This profile will not occur in experiments as there will always be some molecular diffusion. As no mixing occurs, the pdf of density does not change, $\Delta E_{b}=0$, and $\eta=0$. The final profiles are shown in figures $2(\mathrm{a}-\mathrm{c})$ and the pdf of the density profiles are shown in figures $3(\mathrm{a}-\mathrm{c})$. A mixing efficiency of zero is the only value for which there is a unique final density profile as there is only one way in which the system can achieve stability without mixing. For any other value of mixing efficiency, mixing can be combined with sorting to create any of a number of profiles.

We may consider a region as perfectly mixed if its density is uniform; no further mixing is possible in that region without bringing fluid into it from elsewhere. Under this definition, the final state of the classical two-homogeneous layer Rayleigh-Taylor instability may be considered perfectly mixed when $\eta=1 / 2$. For our stratified layers we can extend this idea and pose a second possible final profile where a central turbulent mixing region increases in height until the system is stable and that, when this occurs, this central region is perfectly mixed. Fluid outside the perfectly mixed central region can move but must return to its initial height, leaving the stratification there unchanged. The result is a locally homogeneous region embedded in a profile that is overall stable.

We have denoted this final profile as the perfect mixing profile and its associated mixing efficiency $\eta_{\mathrm{p}}$ (this profile is also described as perfect mixing in Lawrie \& Dalziel (2011a)). We could also have called this the profile of minimum vertical extent as the density profile has changed over the minimum vertical extent to achieve a stable state. The final profiles for this case can be seen in figure $2(\mathrm{~d}-\mathrm{f})$ and the associated pdfs in $3(\mathrm{~d}-\mathrm{f})$. The central peak in the pdf of the final density profile is a delta function. Any spread in this central peak implies some sorting must have occurred in the central mixing region.

The perfect mixing efficiency, $\eta_{p}$ depends on the initial density profile. For the three density profiles shown in figure $2(\mathrm{~d}-\mathrm{f})$, the perfect mixing efficiency for each can be found in table 1. The mixing efficiency for this final state is $\eta_{p}<1$. We can generalise this idea of the perfect mixing profile for any initial density profile $\rho_{0}=\rho(z, t=0)$, stable everywhere apart from an unstable interface at $z=0$. If $\rho_{*}$ is the density in the uniform mixed region that develops as $t \rightarrow \infty$ and $z_{+}, z_{-}$are the upper and lower limits of this region $\left(z_{+}>0>z_{-}\right)$, then conservation of mass can be used to find $\rho_{*}$ as

$$
\int_{z_{-}}^{z_{+}} \rho_{0}(z) \mathrm{d} z=\left(z_{+}-z_{-}\right) \rho_{*} \text { where } \rho_{0}\left(z_{+}\right)=\rho_{0}\left(z_{-}\right)=\rho_{*} \text {. }
$$




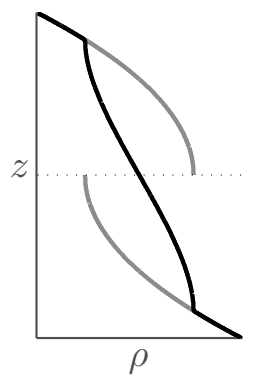

(a)

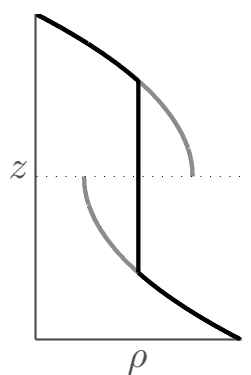

(d)

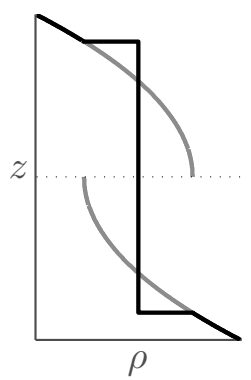

(g)

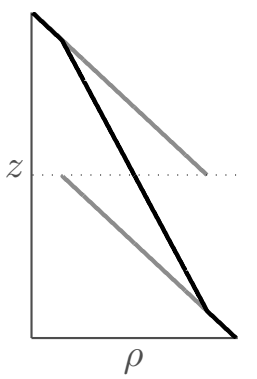

(b)

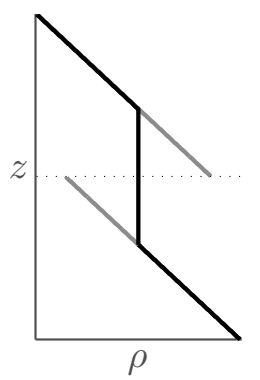

(e)

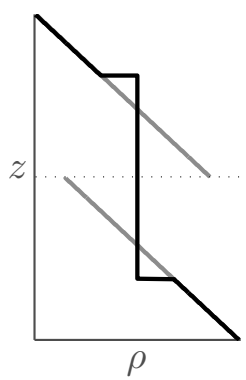

(h)

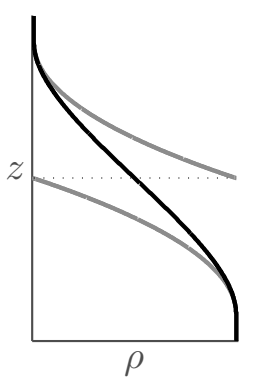

(c)

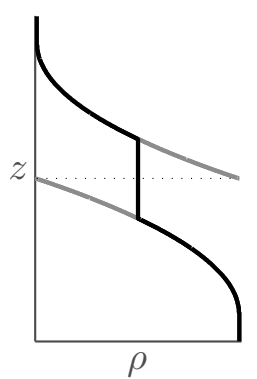

(f)

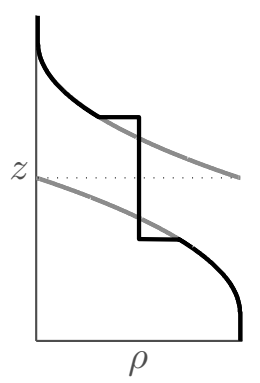

(i)

Figure 2: Possible Final States: Grey lines are initial density profiles, black lines show possible final density profiles, (a-c) sorted density profiles $(\eta=0)$, (d-f) perfect mixing profiles $(\eta=0.682,0.78$ and 0.78$)$, (g-i) examples of $\eta=1$ profiles.

The values of $\rho_{*}, z_{+}$and $z_{-}$define what the perfect mixing profile will be and predict the perfect mixing efficiency for a particular initial density profile.

A third possibility (and the final one we will consider here) is that all the available energy is used in mixing, resulting in $\eta=1$. This situation is not physical as it would require there to be stirring with no viscous dissipation The profile for $\eta=1$ is not unique (indeed there are an infinite number of final profiles with this property), but a possible profile can be constructed as an extension to the idea of the perfect mixing profile. Excess kinetic energy, no longer being dissipated by viscosity, erodes away at the stable profile above and below the central well mixed region, extending this region vertically. This profile can be seen in figures 2 (g-i) and the corresponding pdfs are in figures 3(g-i). There is still a delta function in the pdf at the perfect mixing density as this is the 


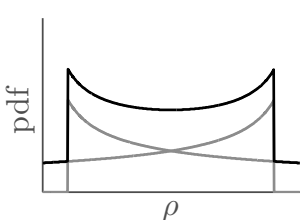

(a)

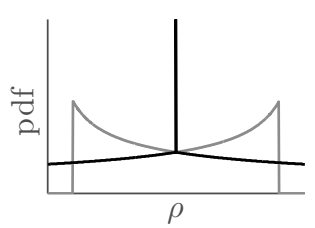

(d)

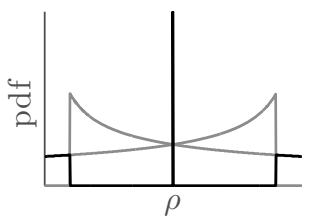

$(\mathrm{g})$

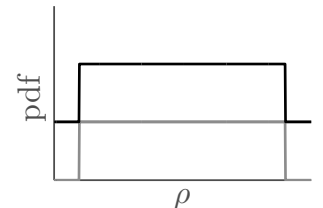

(b)

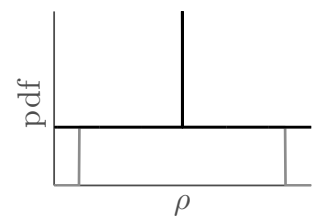

(e)

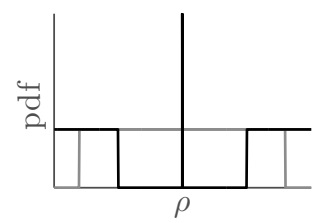

(h)

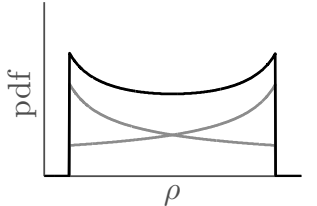

(c)

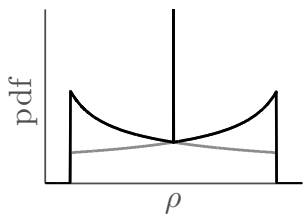

(f)

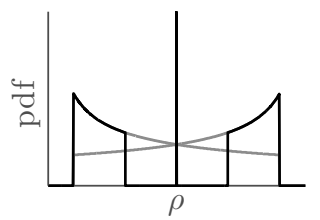

(i)

Figure 3: pdfs of possible final states: Grey lines are the initial layer pdfs, black lines show a possible final density pdf, (a-c) total initial pdf or sorted pdf (the sum of the pdfs of the initial layers), (d-f) perfect mixing pdf, (g-i) possible $\eta=1$ pdf

Figure 2 Profile

(d) Quadratic (with increasing density gradient)

(e) Linear

(f) Quadratic (with decreasing density gradient)
Perfect Mixing Efficiency, $\eta_{\mathrm{p}}$

$$
\begin{aligned}
\frac{\pi-1}{\pi} & \approx 0.682 \\
\frac{3}{4} & =0.75 \\
\frac{3(1+\pi)-8 \sqrt{2}}{3 \pi-8} & \approx 0.780
\end{aligned}
$$

Table 1: Mixing efficiency if the mixing region at the unstable interface is perfectly mixed and grows only until the system is overall stable.

density of the well mixed region. However, around this delta function the pdf is zero as the surrounding densities have been incorporated into the central mixing region.

There are two ways of viewing this final state. If, once the system is stable, the kinetic energy that remains in the mixing region erodes the stable density profiles above and below the mixing region, this erosion could be regarded as a type of external mixing (first defined by Turner (1973)), where turbulence generated elsewhere results in mixing of a stable density profile. If the mixing region can only be extended by this late stage mixing of the stable profiles, outside the turbulent mixing region, then it would appear unlikely that anything like the $\eta=1$ profile described above would be seen in experiments as the mixing efficiency of stable density profiles has been found to be low (e.g. $\eta \sim 0.11$, Prastowo et al. (2008)). Alternatively, mixing of the stable profiles could result from behaviour similar to penetrative convection where a convectively unstable layer erodes a stable stratification (Turner 1973). At this point, the distinction between these scenarios is not critical. Turbulent erosion of a stable interface by RT instability has been previously investigated by Jacobs \& Dalziel (2005) who found the erosion of the stable interface was 


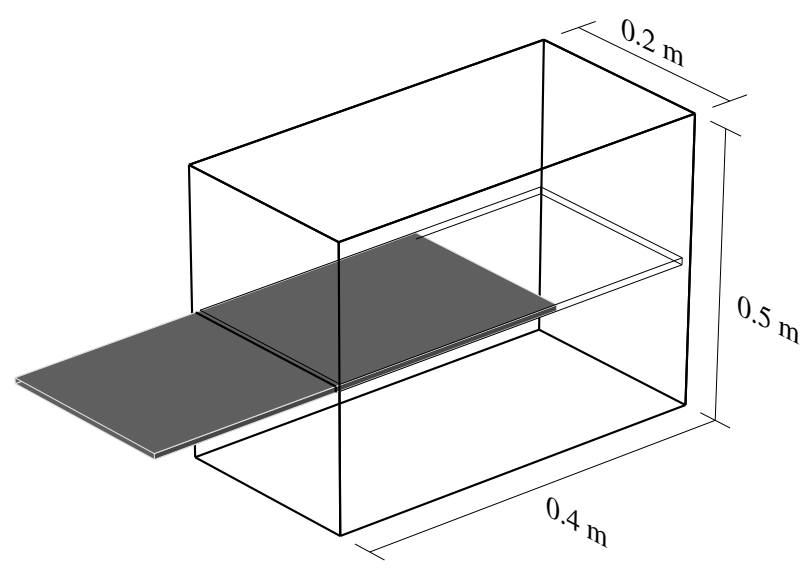

Figure 4: A sketch of the tank with the barrier (shown in grey) half removed.

small and the entrainment rate followed a power law with Richardson number,

$$
R i=\frac{\Delta \rho g l}{\bar{\rho} u^{2}}
$$

where $l$ and $u$ are characteristic length and velocity scales, $\bar{\rho}$ is a characteristic density, and $\Delta \rho$ is the density difference across the interface between the layers.

\section{Experimental setup}

The experimental apparatus is similar to that used by Linden et al. (1994), Dalziel (1993), Dalziel et al. (1999), Jacobs \& Dalziel (2005) and Lawrie \& Dalziel (2011a) (though the barrier used differs from that used in the last four of these studies). In the present work, for any experiment a pair of stable density profiles are set up, one either side of a solid stainless steel barrier, such that the interface between the stable stratifications is unstable to RT instability. The barrier supports the unstable interface while the experiment is being set up. The tank itself is constructed of acrylic and has internal dimensions $0.2 \mathrm{~m} \times 0.4 \mathrm{~m} \times 0.5 \mathrm{~m}$. A sketch of the tank with the barrier slightly less than half removed is shown in figure 4.

Three initial density profiles were investigated, all of the form

$$
\rho(z, t=0)=\rho_{0}(z)= \begin{cases}\left(1+A_{0} f\left(\frac{z}{z_{n}}\right)\right) \bar{\rho}, & \text { for } z \geqslant 0 \\ \left(1-A_{0} f\left(-\frac{z}{z_{n}}\right)\right) \bar{\rho} & \text { for } z<0\end{cases}
$$

where $A_{0}=\frac{\Delta \rho}{2 \bar{\rho}}, \bar{\rho}=\frac{1}{2}\left(\rho_{u}+\rho_{l}\right), \Delta \rho=\rho_{u}-\rho_{l}$, and $\rho_{u}$ and $\rho_{l}$ are the densities of the fluid just above and below the unstable interface. The length-scale $z_{n}$ is the height above (below) the barrier at which $\rho_{0}(z)$ is equal to the density immediately below (above) the barrier (i.e. $f(1)=-f(0)$ ). If there were no mixing then the density profile would change over a vertical height $2 z_{n}$ to achieve stability. The functional forms of the density profiles 
investigated are as follows (expressed in terms of $\hat{z}=\frac{z}{z_{n}}$ ); sketches of these profiles can be seen in figures $1(\mathrm{~b}-\mathrm{d})$.

(a) Quadratic (increasing density gradient, $\frac{\mathrm{d}^{2} \rho}{\mathrm{d}|z|^{2}}>0$ )

$$
f(\hat{z})=1-2 \hat{z}^{2} \text {. }
$$

(b) Linear (constant density gradient, $\frac{\mathrm{d}^{2} \rho}{\mathrm{d}|z|^{2}}=0$ ).

$$
f(\hat{z})=1-2 \hat{z} \text {. }
$$

(c) Quadratic (decreasing density gradient, $\frac{\mathrm{d}^{2} \rho}{\mathrm{d}|z|^{2}}<0$ )

$$
f(\hat{z})=1-4 \hat{z}+2 \hat{z}^{2} \text {. }
$$

The stratified layers were created using a pair of computer-controlled peristaltic pumps, one of which pumped fresh water, the other a solution of $\mathrm{NaCl}$. The two flows were mixed before being pumped into the bottom of the tank though a stainless steel tube. This tube had a hollow cylinder of foam $(2 \mathrm{~cm}$ long) at the exit point to remove the majority of the kinetic energy of the flow. When filling the bottom layer the tube was positioned such that the cylinder of foam rested on the floor of the tank.

The lower layer was filled from the bottom, with the least dense fluid added first, and filling was stopped when the surface was $3 \mathrm{~cm}$ above the height of the barrier. Once the lower layer had been constructed and the density profile measured, the barrier was moved into place and the excess lower layer fluid, now located above the barrier, was removed. The upper layer was then added to the top half of the tank through the filling tube, the end of which was now positioned to be on top of the barrier. The barrier supported the unstable interface between the two layers, while the upper layer was being added. Once the upper layer was filled, the density profile of the upper layer was measured, approximately an hour after the density profile of the lower layer was measured and five minutes before the barrier was removed. The final density profile was measured twenty minutes after the barrier was withdrawn (by which time all visible motion had decayed).

An aspirating conductivity probe, constructed in-house, combined with a fast response thermistor (General Electric type NTC P25) was used to measure the initial and final density profiles. For each measurement of the density profile, the probe was traversed downwards at a speed of $4 \mathrm{~mm} \mathrm{~s}^{-1}$ and conductivity measurements recorded at a frequency of $50 \mathrm{~Hz}$. The temperature measurement was important for the accurate measurement of density. The mixing of the two layers results in the release of a heat of dilution (which can be either positive or negative over the values of density considered) and there is evaporative cooling at the surface of the fluid layers. This thermal signal has a negligible direct effect on the density (via thermal expansion), but a small effect on the conductivity for a given salinity, necessitating the calibration of the conductivity probe with both conductivity and temperature in order to measure the density profiles to the desired accuracy. The accuracy of the probe is limited by electronic noise, introducing a random error in the density measurement at a given point of $7 \times 10^{-5} \mathrm{~g} / \mathrm{cm}^{3}$ which is $0.2 \%$ of the range of $\rho$ measured by the probe.

Aspiration of the probe removes a measurement volume of $18 \mathrm{ml}$ for every $0.5 \mathrm{~m}$ that is traversed. This equates to a volume of $3 \mathrm{~mm}^{3}$ per sample, where a sample is the density measurement at a particular height. A single traverse will take up to 6250 samples (when the entire depth of the tank is traversed). The density of a sample will be the density averaged across this volume (and therefore the density averaged across a finite height, $\Delta z$ ). Where there is a strong density gradient, the majority of this measurement volume will be drawn from the current height of the probe tip (reducing the error in measurement 
height). Where there is a weak stratification the volume may be taken from just above and below the measurement position (at most $\pm 0.7 \mathrm{~mm}$ ) but as the density variation is necessarily smaller in this case the resultant error is still small.

The barrier that separates the unstable interface before the start of an experiment is removed by a motor at a speed of $U=0.08 \mathrm{~m} \mathrm{~s}^{-1}$. The composite barrier used in other studies was constructed so as to reduce the shear between the barrier and the fluid, but unavoidably results in more leakage from the tank. Using a solid barrier (similar to that used by Linden et al. (1994)) it was possible to achieve a far better seal on the tank, eliminating the errors in calculating the potential energy which stem from fluid being lost in this way from the tank over the course of an experiment. The addition of a motor (not used in any of the previous studies) substantially improved the repeatability of the barrier removal. It is clear that the barrier will have a significant effect on the initial conditions of a flow. However, it is not possible to set up the configurations required using an accelerated box, magnetic fields or overturning of a stable situation, as the density profiles we are investigating are unstable to acceleration in both the upwards and downwards directions.

The effect of the barrier on the initial conditions of the flow can be split into three contributions. Firstly, there is a potential energy change associated with the downward movement of the upper layer. This dominates all other contributions but is exactly balanced out by the work done on the end of the barrier by the hydrostatic pressure as the barrier is removed and may therefore be ignored (Dalziel 1994). Secondly, kinetic energy is added to the flow due to the formation of viscous boundary layers above and below the barrier. This contribution scales as $U^{\frac{3}{2}}$ and for these experiments is a maximum of $3 \%$ of the initially available potential energy (the calculation of this is detailed below). Thirdly, there is an addition of kinetic energy associated with non-hydrostatic pressure fluctuations at the end of the barrier due to the removal of a finite volume. This scales as $U^{2}$ and for these experiments is $O\left(10^{-4}\right) \%$ of the initial $E_{a}$. Although the magnitude of this contribution is small it has an effect on the structure of the initial conditions as it results in a jet down the right-hand side-wall of the tank. Experiments were performed to investigate the effect of this jet (these are detailed below).

A linear approximation for the kinetic energy that the barrier adds to the flow through the formation of a viscous boundary layer can be made by assuming that the Rayleigh layer that forms above and below the barrier, due to velocity being diffused vertically by viscosity, has the profile $u(z, t)=U \operatorname{erfc}\left(\frac{|z|}{2 \sqrt{\nu t}}\right)$ (where $U$ is the velocity of the barrier and $\nu$ is the kinematic viscosity). If we ignore end effects, the total kinetic energy input is equal to

$$
\begin{aligned}
E_{k} & =\left.2 \nu \bar{\rho} L W U \int_{0}^{T} \frac{\partial u}{\partial z}\right|_{z=0} d t, \\
& =\frac{8}{3} \sqrt{\frac{\nu}{\pi}} W \bar{\rho}(U L)^{\frac{3}{2}},
\end{aligned}
$$

where $W$ is the width of the barrier, $L$ is the length of the barrier, and $T=\frac{L}{U}$ the time taken to withdraw the barrier. For the experiments presented in this paper, $E_{k}$ was less than $3.06 \%$ of the initially available potential energy (for the lowest Atwood number cases) and was less than $1.5 \%$ of $E_{a}(t=0)$ for $A_{0}>0.006$. A naive assumption might be to expect the mixing efficiency calculated purely from measured density profiles (i.e. $\eta_{R T}$ ) to be around $3 \%$ more than the true mixing efficiency (as we are adding $3 \%$ more available energy than we have accounted for in our calculation of mixing efficiency). However, the barrier adds a two-dimensional perturbation to the flow at a wavelength 
set by the instability in the barrier wake. This can be see in figure 5a where a regular wavelength appears on the surface of the mixing region as the barrier is removed. Here the flow was visualised by dyeing the lower layer with red and blue food dye, along with the fluorescent green dye fluorescein disodium. The upper surface of the mixing region is illuminated with halogen lamps. A video of the instability using the same method for visualization was submitted by the authors to the APS gallery of fluid motion in 2012 and a similar video is included with this paper as supplementary material. A similar series of images for the classical two-layer case can be seen in Dalziel et al. (1999).

The wavelength introduced by the instability of the barrier's wake breaks down quickly in the presence of RTI, leaving the three-dimensional bubbles shown in figure 5b. The finite wavelength at which the barrier adds kinetic energy influences the barrier's effect on the mixing efficiency. This will be discussed further in $\S 4$.

When the barrier exits the tank the viscous boundary layer is stripped off. Two strong vortices are created, one above and one below the slot where the barrier exits the tank. This injects kinetic energy into the flow as the shear stress here will be greater than over the rest of the barrier. This vortex can be seen at the left hand side of figure $5 \mathrm{~b}$. The energy of this is included in the calculation in (3.6), though the length-scale of this vortex is much larger than the wavelength introduced by the instability in the barrier wake.

For a standard experiment, the removal of the finite volume of the barrier results in a downward jet on the right-hand side-wall of the tank. The upper layer can move down to fill the void left by the barrier, but the lower layer cannot move (as it does not have a free surface). The result is the formation of a Kutta condition and separation region at the trailing edge of the barrier. A potential flow solution for this contribution to the kinetic energy (with experimentally derived coefficients) is descried in Dalziel et al. (1999). At the barrier speeds used in this investigation the contribution from the finite volume removal is small $\left(O\left(10^{-4}\right) \%\right.$ of the initial $\left.A P E\right)$. To assess the effect of this, some experiments were performed where, as the barrier was removed, fluid was pumped into a bag placed at the bottom of the tank. This was an attempt to reverse the initial conditions generated by the volume removal, so that the jet formed on the right hand wall would be upwards rather than downwards. While we succeeded in reversing this jet, no significant differences were found in the shape of the final profile or in the value of the mixing efficiency, though the error in these experiments was much higher. This increased error was due to the high degree of accuracy required for the measurement of the fluid being pumped into the base (as this represents a relatively large addition of potential energy).

\section{Observations and measurements}

\subsection{Development of the mixing region}

In the classical case of two RT unstable layers, each of constant density, the growth of the turbulent mixing region has several regimes: (i) linear stability analysis shows that initially small perturbations grow exponentially; (ii) when the amplitude of the most unstable wave reaches a magnitude comparable with half its wavelength, growth becomes non-linear; (iii) a non-linear growth phase which will be self-similar if all memory of initial conditions are lost (Ramaprabhu \& Andrews 2004). The growth of the instability continues to accelerate until the mixing region fills the domain of the experiment.

In the stratified case, the initial behaviour of the instability is similar to the classical case, but rather than continuing to accelerate, the stable stratification begins to reduce and eventually reverses the acceleration, slowing the growth of the mixing region. The 


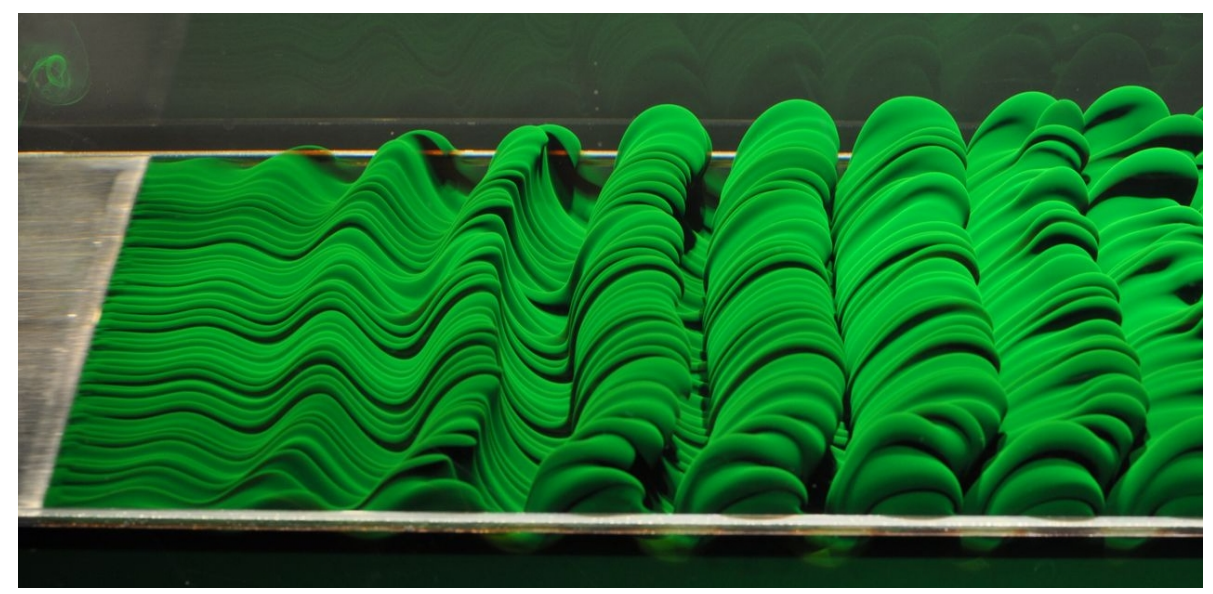

(a)

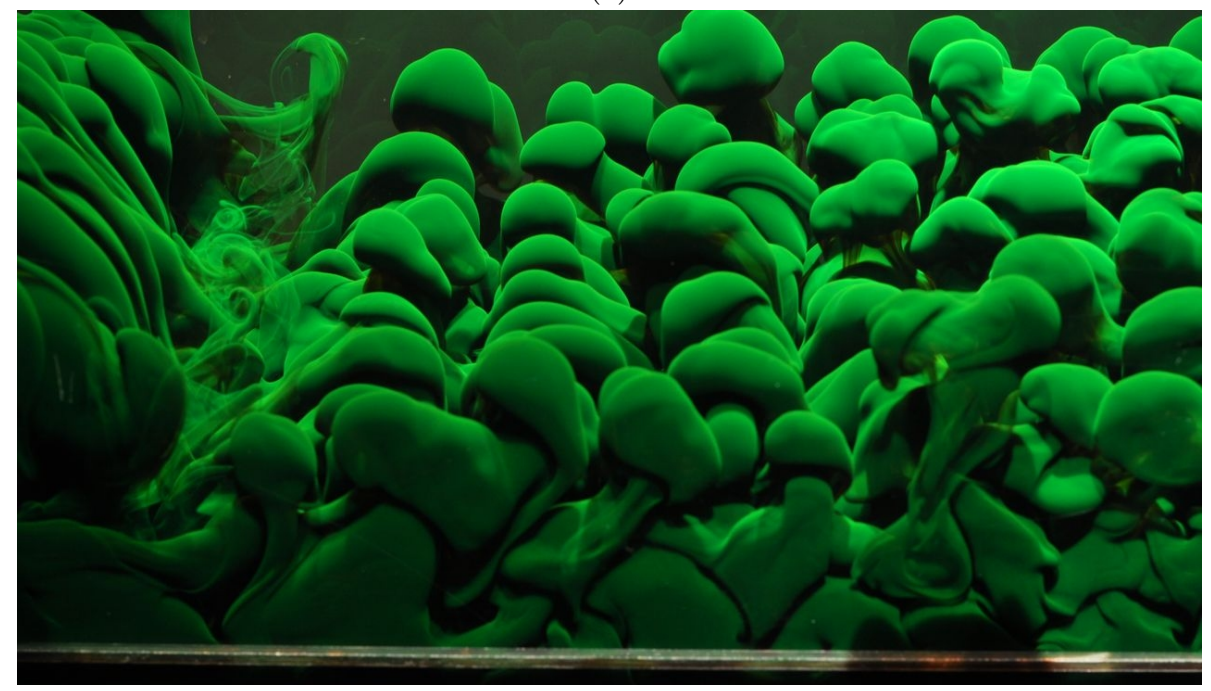

(b)

Figure 5: Photograph showing the upper surface of the mixing region when (a) $t=4 s$, the barrier is in the process of being removed and (b) $t=11 s$, the mixing region has developed a three-dimensional structure. A vortex introduced by the barrier is visible on the left hand side of the image. The barrier starts moving at $t=0$ at the standard withdrawal speed. The lower layer of the tank was dyed with red, blue and fluorescent green dye for this visualisation. This experiment was at a much lower Atwood number than other experiments, $A_{0}=7 \times 10^{-4}$. This view was taken with the camera looking slightly down at the upper layer.

development of the instability, when it is confined by stratification, is shown in the shadowgraph images of figure 6 . This experiment had an initially quadratic density profile with a density gradient which increased away from the unstable interface (figure 1b shows a sketch) and an Atwood number of $A_{0}=9 \times 10^{-3}$. The times quoted for this figure are non-dimensional, $\tau=\sqrt{\frac{A_{0} g}{z_{n}}} t$.

When the barrier is removed, a turbulent mixing region develops at the unstable in- 


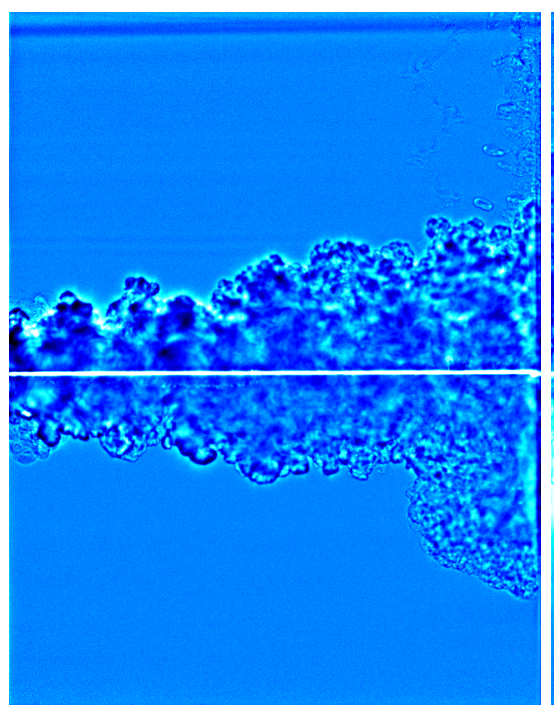

(a)
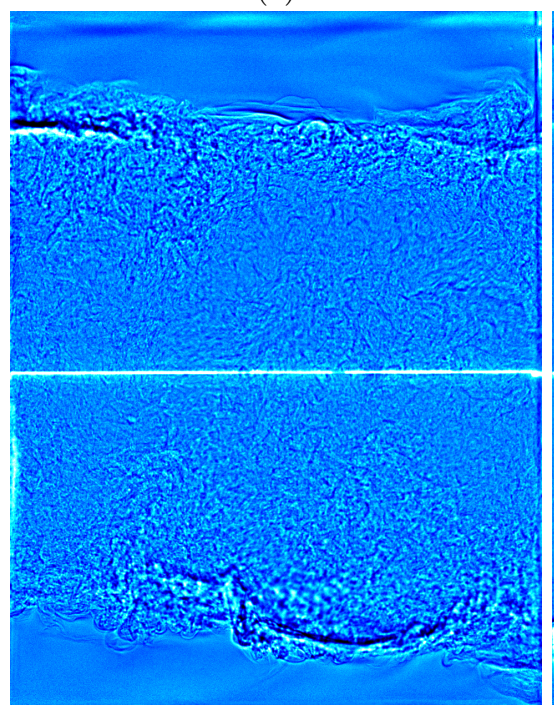

(c)

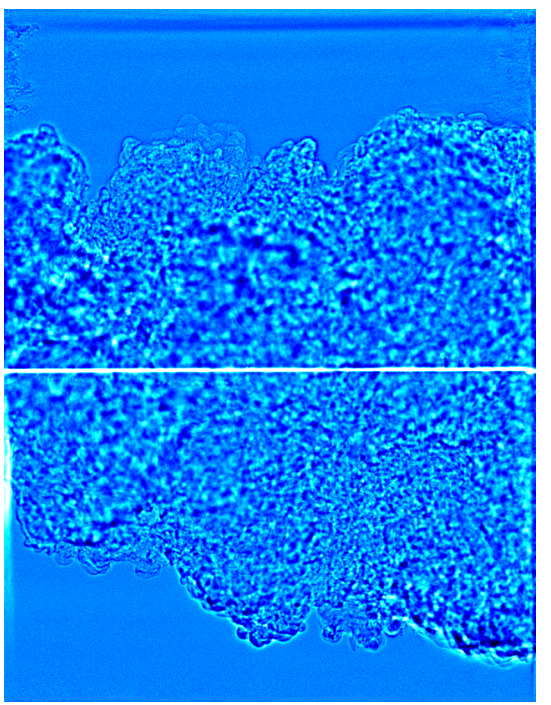

(b)

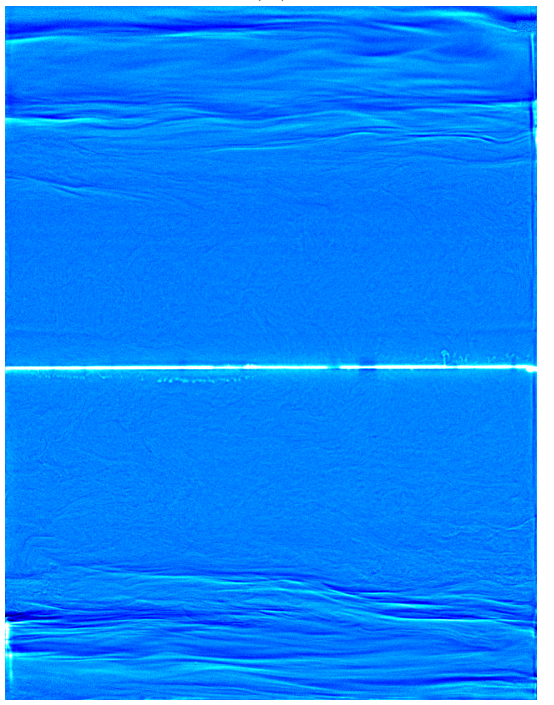

(d)

Figure 6: Shadowgraph images showing the development of the instability for $A_{0}=$ $9 \times 10^{-3}$, (a) $\tau=3.3$, a mixing region develops at the unstable interface; (b) $\tau=6.6$, stable stratification slows the growth of the mixing region; (c) $\tau=13.3$, turbulent mixing continues but there is no further growth of the mixing region; (d) $\tau=53.2$, motion has died away in the central region but internal waves remain in the stratification above and below this region. These images are of the side of the tank and are integrated views across the tank. The horizontal white line through the centre of each image is the original location of the barrier.

terface. Figure 6a $(\tau=3.3)$ shows the flow when the barrier has just exited out of the left hand side of the tank. The turbulent mixing region grows with time into the stablystratified layers above and below. The stratification initially accelerates the growth of the mixing region, but eventually this acceleration slows and the stratification begins to 
decelerate the growth of the mixing region, eventually arresting its growth as the profile becomes stably stratified. The vortex due to the stripping the boundary layers from the barrier (as discussed in §3) is shown to be relatively weak in this higher $A_{0}$ experiment (compared to figure 5b), as it does not produce a visible signal.

As the stratification has an increasing density gradient, the growth decelerates faster the further the mixing zone extends. In figure $6 \mathrm{~b}(\tau=6.6)$ the mixing region has reached its final extent on the right hand side of the tank, while elsewhere in the flow the height of the mixing region is smaller and still increasing. Although the flow is clearly still turbulent and large scales are present, confinement by the stratification is inevitable. By $\tau=13.3$ (figure 6c) the mixing region has reached its final extent throughout the tank and further growth is inhibited by the stable stratification. The mixing region still appears to be turbulent with fine-scale mixing occurring. The reduced texture of the shadowgraph indicates that density differences within the mixing region are greatly diminished. By figure $6 \mathrm{~d}(\tau=53.2)$, density differences in the central mixing region have been smoothed out, leaving internal waves in the remaining stratified zones above and below the central region. These internal waves have small amplitudes and long wavelengths (comparable with the width of the tank), which contribute to the dissipation of the remaining energy. For the experiment shown in figure 6 , the energy contained in the internal waves outside the mixing zone is estimated to be less than $0.2 \%$ of the initial available potential energy.

The development of structures on the surface of the mixing region can be seen in figure 7 , which uses the same visualization method as figure 5 (described in §3). As with the experiment shown in figure 6 , this experiment had an initially quadratic density profile with an increasing density gradient $\left(A_{0}=2 \times 10^{-3}\right)$. This method of visualization allows us to observe (figure $7 \mathrm{a}, \tau=4.1$ ) the progression of bubbles of low density fluid (from the lower layer), as they rise up though the stable stratification in the upper layer. The length-scale of these bubble-like structures scales with the height of the mixing region, therefore they increase in size as they grow into the upper layer. Similar structures from the upper region descend into the lower layer.

By $\tau=9.1$ (figure $7 \mathrm{~b}$ ) further vertical growth of the mixing region has been arrested on the right hand side of the tank by the stable stratification. A range of scales can be observed, from large scale bubbles that span the height of the mixing region to small bubbles on scales $\sim O(4 \mathrm{~mm})$. When compared with figure $7 \mathrm{a}$, there has been a flattening of structures due to the effects of the stable stratification. By $\tau=13.4$ (figure 7c) bubblelike structure of the top of the mixing region have disappeared and the mixing region appears homogeneous, although internal waves remain on the upper surface of the mixing region. Wisps of green fluid appear just above the central mixing region, showing that a small amount of fluid that originated in the lower layer ends up outside the central mixing region. Long term behaviour cannot be observed as the arc lamps used for this visualisation heat the dyed fluid from the lower layer more than the clear fluid from the upper layer. This results in a double diffusive instability at late times or if the Atwood number is reduced further.

\subsection{Final density profiles}

The initial and final density profiles for three typical experiments, one for each of the profiles investigated, are shown in figures 8 to 10. Many more than three experiments were performed (more than 150) and these profiles were found to be repeatable. The final state in experiments was found to be similar to that defined as the perfect mixing profile (see figures 2(d-f)): there is a central well-mixed region of almost constant density, surrounded by stable stratifications. This surrounding stable stratification is nearly unchanged from the original profile. 


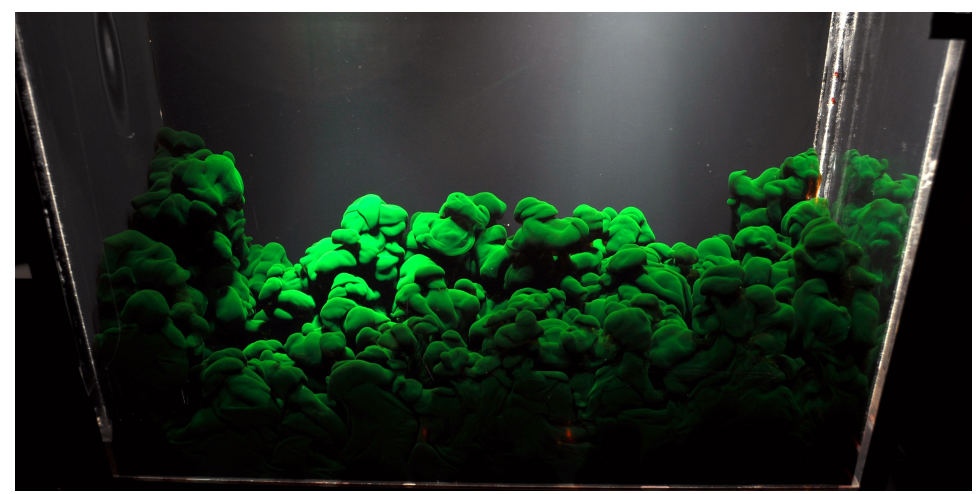

(a)

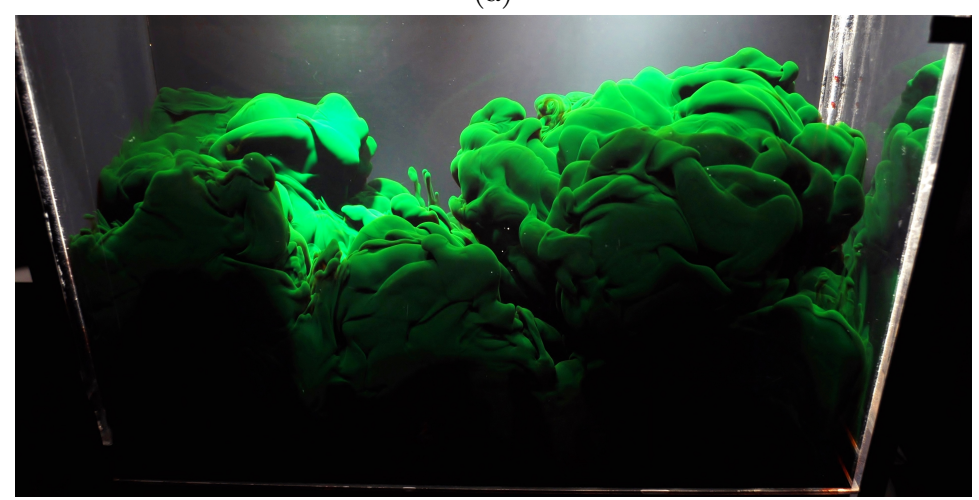

(b)

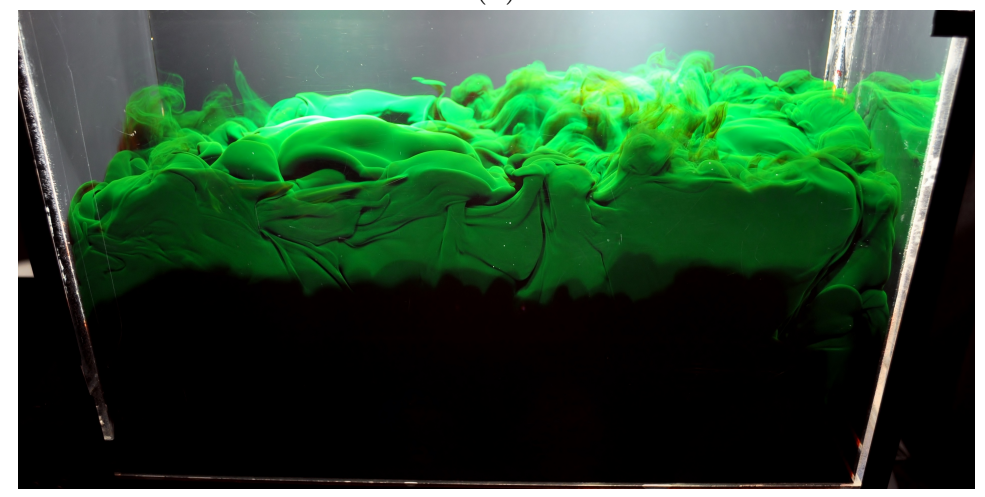

(c)

Figure 7: Photographs showing the mixing region being confined by the stable density stratification: (a) $\tau=4$.14, large-scale structures extend upwards into the mixing region from the lower layer; (b) $\tau=9.07$, the stratification has arrested further vertical growth of the mixing region; (c) $\tau=13.37$, motion has decayed, except for internal waves present on the upper surface of the mixing region. $A_{0}=2 \times 10^{-3}$, initial stratification was quadratic with increasing density gradient. This view was taken with the camera looking slightly down at the upper layer. 


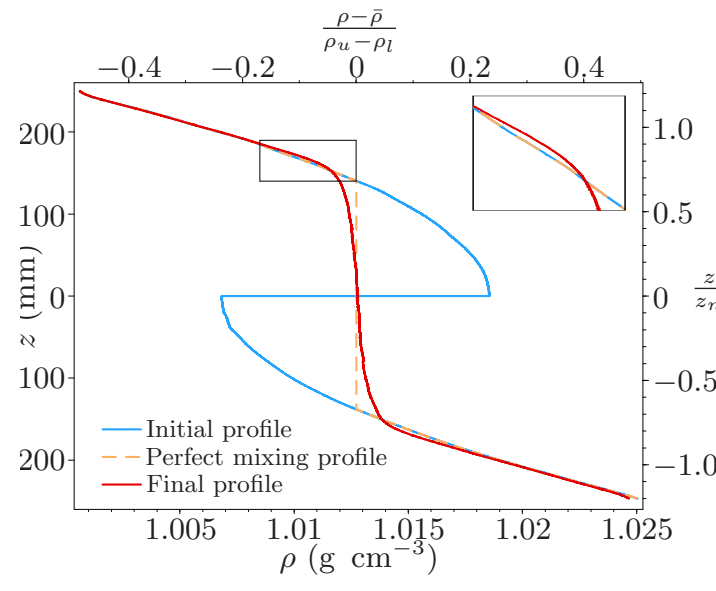

(a)

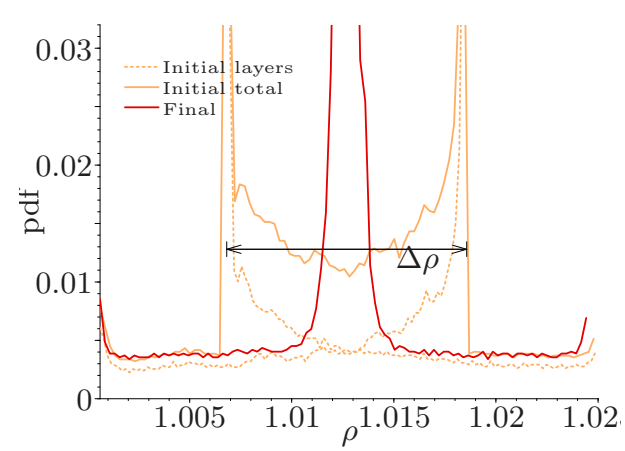

(b)

Figure 8: Quadratic (increasing density gradient) initial density profile: (a) Density profiles and (b) pdf of density. $A=5.80 \times 10^{-3}$ and $\eta_{R T}=0.657 \pm 0.004 .\left(\eta_{\mathrm{p}}=0.682\right.$, as calculated from $\rho_{0}$.)

The mixing efficiency of all these experiments was close to the perfect mixing efficiency, as calculated from the initial density profiles. For the quadratic case with increasing density gradient, the mixing efficiency was $0.657 \pm 0.004\left(=0.96 \times \eta_{\mathrm{p}}\right)$, in the linear case the mixing efficiency was $0.741 \pm 10^{-4}\left(=0.99 \times \eta_{\mathrm{p}}\right)$ and in the quadratic case with decreasing density gradient the mixing efficiency was $0.759 \pm 10^{-4}\left(=0.98 \times \eta_{\mathrm{p}}\right)$.

In all experiments there was some sorting within the central region, a process that reduces the mixing efficiency compared with perfect mixing. In some cases this was partly offset by a slight alteration of the stable profiles outside the central region, a feature that contributes to an increased mixing efficiency. We can see examples of the latter in figures $8 \mathrm{a}$ and $9 \mathrm{a}$.

We wish to quantify the effect on the mixing efficiency of sorting where the fluid could have mixed to become stable (i.e. in the central region), which decreases the mixing efficiency, with mixing of the stable density profiles outside the central mixing region (where mixing is not necessary for stability) which increases the mixing efficiency. We can quantify the effect of the mixing of the stable profiles by comparing the potential energy increase above and below where the final density profile crosses the perfect mixing profile. Similarly, we can quantify the amount of sorting within the central region by comparing the potential energy of the central region (between the points in the upper and lower layers where the final density profile crosses the perfect mixing profile) to the potential energy that the perfect mixing profile would result in.

In the experiment shown in figure $8 \mathrm{a}$, the mixing of the stable profile above and below the central mixing region increases the mixing efficiency by $2.3 \%$ and the residual stratification within the central region reduces the mixing efficiency by $4.8 \%$ compared to the perfect mixing value. In contrast, the alteration of the stable profiles does not appear in figure 10a, where we can see a residual stratification in the central region but no signifi- 


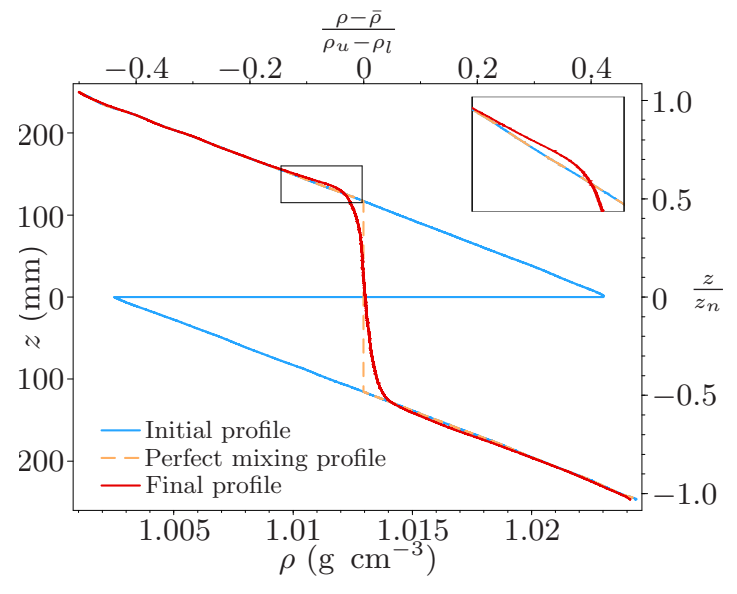

(a)

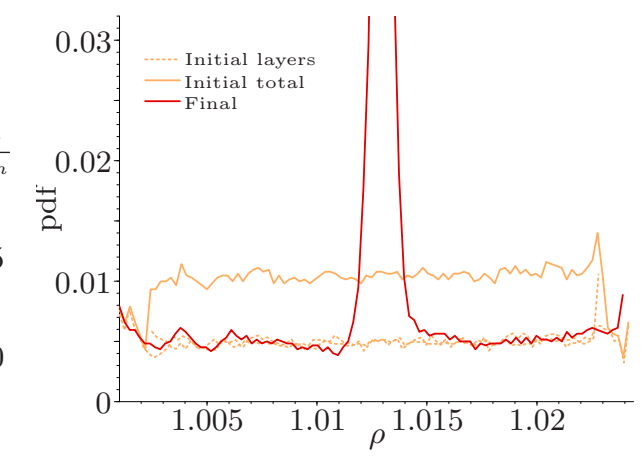

(b)

Figure 9: Linear (constant density gradient) initial density profile: (a) Density profiles and (b) pdf of density. $A=1.01 \times 10^{-2}$ and $\eta_{R T}=0.741 \pm 10^{-4} \cdot\left(\eta_{\mathrm{p}}=0.75\right.$, as calculated from $\rho_{0}$.)

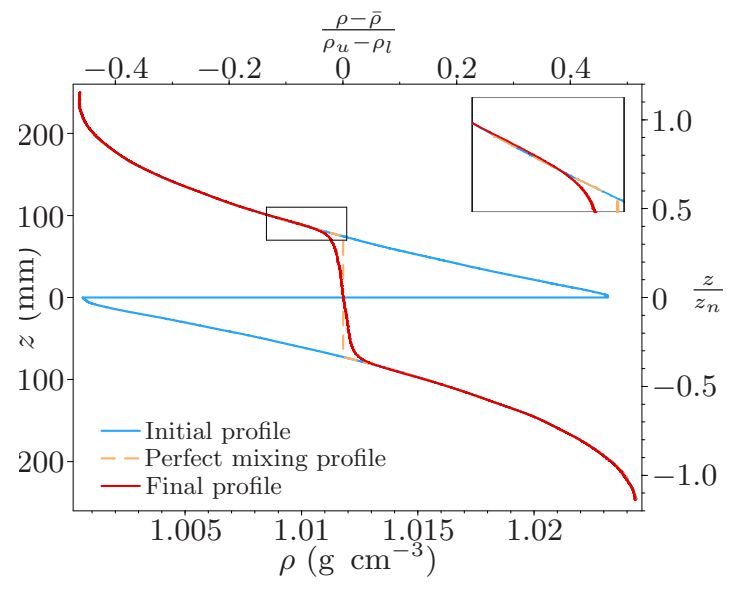

(a)

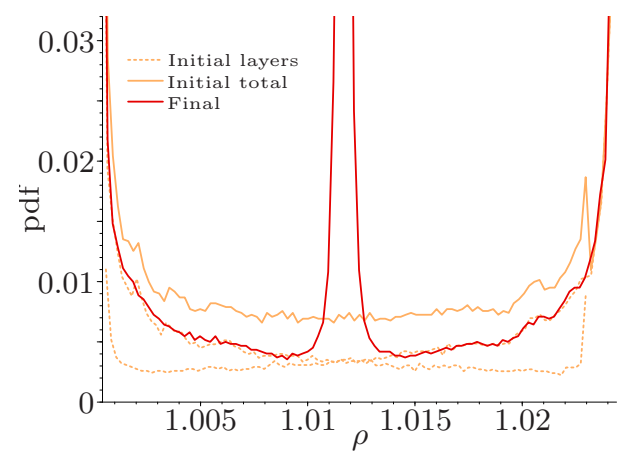

(b)

Figure 10: Quadratic (decreasing density gradient) initial density profile: (a) Density profiles and (b) pdf of density. $A=1.11 \times 10^{-2}$ and $\eta_{R T}=0.759 \pm 10^{-4} \cdot\left(\eta_{\mathrm{p}}=0.777\right.$, as calculated from $\rho_{0}$.) 
cant mixing in the stable profiles outside the mixing region. This residual stratification reduces the mixing efficiency by $1.8 \%$ (as compared to the perfect mixing profile).

The pdfs of density for these experiments are shown in figures $8 \mathrm{~b}, 9 \mathrm{~b}$ and $10 \mathrm{~b}$. If the density in the central mixing region were constant (perfectly mixed), then the peak in the pdf would be a delta function as shown in figures $2(\mathrm{~d}-\mathrm{f})$. The spread of the peak in the pdf for each experiment is a measure of the sorting that has occurred in the central mixing region.

We can relate the mixing of the stable profiles outside the central mixing region to the gradient Richardson number, as measured across the edge of the mixing region, towards the end of the development of the flow. The gradient Richardson number is defined as

$$
R i_{e}=\frac{N^{2}}{(\mathrm{~d} u / \mathrm{d} z)^{2}},
$$

where $N$ is the Brunt-Väisälä or buoyancy frequency, $N^{2}=-\frac{g}{\rho} \frac{\mathrm{d} \rho}{\mathrm{d} z}$ and $u$ is a velocity. Mixing here is driven by potential energy released from the unstable density profile. Let us assume that the kinetic energy available for mixing the stable profiles,

$$
\frac{1}{2} V \bar{\rho} u^{2} \sim \Delta E_{p}
$$

where $\Delta E_{p}$ is the change in gravitational potential energy of the density profile between the initial state and the corresponding perfect mixing profile and $V$ is the volume of the mixing region $\left(V=W L z_{p}\right.$, where $z_{p}$ is the height of the perfect mixing region and $W$ and $L$ are the width and length of the tank). This gives us a way of estimating how the turbulent velocity scale $u$, just inside the mixing region, will change for profiles with different $\Delta E_{p}$ and $z_{p}$. There is little motion outside the mixing region (compared to the turbulence within it), therefore if the mixing of stable profiles is occurring in a thin layer that has thickness $\delta$, then we can assume that the velocity gradient across this layer scales like $\frac{\mathrm{d} u}{\mathrm{~d} z} \sim \frac{u}{\delta}$. We can use these arguments to find $R i_{e}$ for each density profile. For example, in the linear density profile case, $\Delta E_{p}=\frac{1}{24} g z_{n}^{2} \Delta \rho W L$, the height of the perfect mixing region $z_{p}=\frac{z_{n}}{2}$ and $\frac{\mathrm{d} \rho}{\mathrm{d} z}=-\frac{\Delta \rho}{z_{n}}$. Putting all this into equation (4.1) we find that $R i_{e} \sim 6 \frac{\delta^{2}}{z_{n}^{2}}$. For the quadratic with increasing density gradient $R i_{e} \sim 4 \frac{\delta^{2}}{z_{n}^{2}}$ and for the quadratic with decreasing density gradient $R i_{e} \sim \frac{6(\sqrt{2}-1)}{(8 \sqrt{2}-11)} \frac{\delta^{2}}{z_{n}^{2}} \approx 7.9 \frac{\delta^{2}}{z_{n}^{2}}$ at the perfect mixing height.

Another way of viewing this is if mixing around the central region occurs at some critical Richardson number $R i_{c}$, then we would expect the distance across which mixing occurs $\delta$ to be different for each of the profiles considered. If $R i_{e} \sim R i_{c}$, then $\delta \sim$ $\sqrt{\frac{R i_{c r i t}}{c}} \times z_{n}$, where $c$ is a different constant for each of the profiles considered $(c=4$, 6 and 7.9 for the quadratic with increasing density gradient, linear and quadratic with decreasing density gradient, respectively). If $c$ is larger, we expect the region where mixing occurs in the stable regions outside the central mixed region to be smaller and this is what is observed in experiments.

These results show that mixing of the stable profiles occurs to a greater degree in density profiles where the gradient Richardson number is lower as the density profile approaches stability. This behaviour was also found by Jacobs \& Dalziel (2005), where mixing of a stable interface by turbulence generated in a Rayleigh-Taylor unstable region was found to be a function of Richardson number. 


\subsection{Effect of barrier speed on mixing efficiency}

Until now we have been using $\eta$ interchangeably with $\eta_{R T}$ (defined in $\S 2$ ). In using $\eta_{R T}$ we have assumed that the initial conditions are quiescent (or rather that $\Delta E_{k}=0$, indicating there is no change in kinetic energy between the initial and final states). As noted in $\S 3$, the barrier introduces kinetic energy into the initial conditions, therefore the underlying mixing efficiency, $\eta \leqslant \eta_{R T}$ (in calculating $\eta$ we must include in the denominator the small contribution from kinetic energy that was neglected in $\eta_{R T}$ ).

To investigate the effect of barrier withdrawal speed (and hence $\Delta E_{k}$ ) on the mixing efficiency, a series of experiments were performed where the barrier speed was varied from $0.02 \mathrm{~m} / \mathrm{s}$ to $0.8 \mathrm{~m} / \mathrm{s}$. If we add kinetic energy $\left(E_{k}\right)$ into the system in the initial conditions then we might expect this extra energy to do some mixing and increase the change in background potential energy. When we calculate $\eta_{R T}$ we assume the initial conditions are quiescent. In other words, when we add $E_{k}$ we expect more mixing (which increases the numerator of $\eta_{R T}$ ) but we have not accounted for the extra energy in the system (by increasing the denominator). This means we would expect $\eta_{R T}$ to increase when the barrier is withdrawn faster.

Contrary to expectations, we find that the total mixing achieved decreases when the barrier is withdrawn faster, indicating that both the mixing efficiency calculated under the assumption of quiescent initial conditions, $\eta_{R T}$, and the one taking into account the initial kinetic energy, $\eta$, are decreased by faster withdrawals. When the barrier speed was increased, the final profile had a more strongly stratified central region, indicating that additional sorting had occurred. The extra kinetic energy available at the start of the instability is at finite length-scales and appears to be preferentially sorting the profile, rather than mixing it. This effect is more significant at barrier speeds less than $0.08 \mathrm{~m} / \mathrm{s}$. As it is desirable to keep the spatial variation of the instability to a minimum, a barrier speed of $0.08 \mathrm{~m} / \mathrm{s}$ was used for all experiments described here.

\subsection{Effect of Atwood number on mixing efficiency}

To investigate the effect of Atwood number on mixing efficiency, a set of twenty-six experiments with linear profiles and Atwood number varying from $4 \times 10^{-3}$ to $7 \times 10^{-2}$ was performed (figure 11). As all these experiments were performed in the same apparatus with the same functional form for the density profile, this range of Atwood numbers spanned Reynolds numbers over the range $R e=1000$ to 4200 . Here the Reynolds number is defined as the maximum of $R e=\frac{h \dot{h}}{\nu}$ (where $h$ is the height of the mixing region and $\nu$ is kinematic viscosity) using the model for $h$ which will be described in $\S 5$. The mixing efficiency of these experiments was always at least $95 \%$ of the perfect mixing efficiency, $\eta_{\mathrm{p}}=0.75 \pm 0.002$ (where $\eta_{\mathrm{p}}$ was calculated from the measured initial density profile of each experiment).

The ratio of the height of the stratification relative to the height of the tank was one of two values in these experiments. Most of the experiments were with $z_{n} \approx \frac{2}{5} H$, but some have $z_{n} \approx \frac{1}{2} H$ (where $H$ is the total height of fluid in the tank). This was found to have little effect on the mixing efficiency so long as the final height of the mixing region was much less than the height of the tank and $z_{n} \leqslant \frac{1}{2} H$.

These experiments show an increase in mixing efficiency with Atwood number, although there is some uncertainty as to whether this increase is due directly to Atwood number or to the dependence of Reynolds number on $A_{0}$. As we saw in the previous subsection, increasing the kinetic energy introduced by the barrier lead to a greater degree of sorting in the central mixed region. It is therefore conceivable that the decrease in $\eta$ at lower $A_{0}$ is due to the increased relative importance of this kinetic energy compared with 


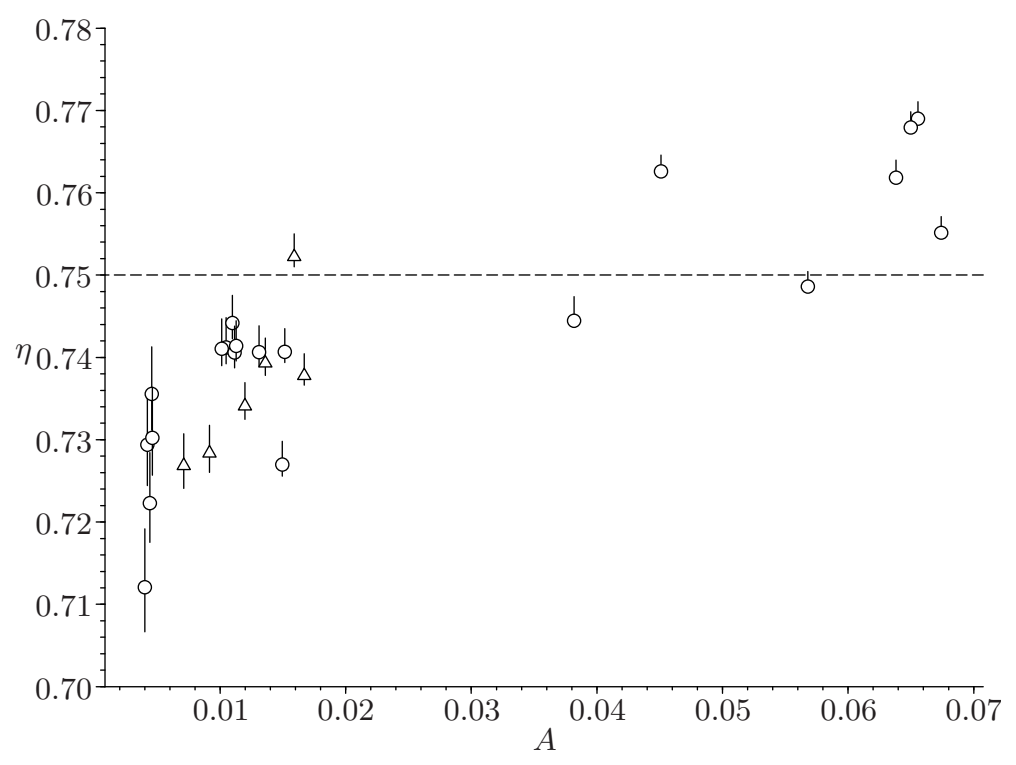

Figure 11: Mixing efficiency of experiments with linear stratifications in the upper and lower layers. $z_{n} \approx \frac{2}{5} H(\bigcirc), z_{n} \approx \frac{1}{2} H(\triangle)$. Dashed line shows perfect mixing efficiency $\eta_{\mathrm{p}}$, which for all experiments was $0.75 \pm 0.002$. All experiments had a mixing efficiency of at least $0.95 \times \eta_{\mathrm{p}}$.

the initially available potential energy, the ratio of which scales (for a given functional form of the density profile) as $\frac{\sqrt{\nu}}{g} \frac{U^{\frac{3}{2}}}{A_{0} z_{n}^{2}}$.

When the effect of the barrier is expected to be small (i.e. the higher Atwood number experiments) the mixing efficiency is higher than the perfect mixing efficiency $\eta_{p}$. In these experiments the mixing of the stable profiles outside the central well-mixed region increases the mixing efficiency beyond the perfect mixing value.

There is more variation at very low Atwood numbers as many of the effects that result in variation between experiments are more significant. For example, the relative magnitude of the kinetic energy of the barrier, as compared with the initially available potential energy, is much greater for these experiments ( $3 \%$ of $A P E$ rather than $\sim 1 \%$ ). The signal to noise ratio for the lower Atwood number experiments $(A<0.01)$ and the mid-range Atwood number experiments $(0.03<A<0.05)$ is larger. Noise in the signal from the conductivity probe is a greater proportion of the density range that is being measured, fluctuations are $0.8 \%$ of the range of $\rho$ rather than $0.2 \%$ for experiments with $0.01<A<0.03$. The mid-range Atwood number experiments are affected as the probe was recalibrated for a higher density range for $(A>0.03)$. The signal to noise ratio is also large in the very high Atwood number experiments $(A>0.06)$ as the gradient of conductivity with density is smaller when the salt solution nears saturation. Therefore noise in the signal has a greater effect at these Atwood numbers. 


\section{Model for the height of the mixing region}

\subsection{Turbulent diffusion model}

We will now construct a model for the height of the mixing region, under the assumption of self-similarity, for the case where the unstable density interface is surrounded by stable stratifications. This is a stratified version of the model described in the introduction $\left(h=\alpha A_{0} g t^{2}\right.$ ). We will begin with a buoyancy-drag model (following Dalziel et al. (2008) and similar to Cook et al. (2004)) and assume an instantaneous balance between the two forces present in the flow, buoyancy and inertia,

$$
\Delta \rho g l \sim \bar{\rho} u^{2} .
$$

Here $\Delta \rho$ is a typical density difference over a distance $l$, the buoyancy length-scale, $u$ is an inertial velocity scale, and $\bar{\rho}$ is the mean density in the flow. We assume that the buoyancy length will scale with the height of the turbulent mixing region $l \sim h$, making $\Delta \rho$ the density difference across the mixing region, $\Delta \rho=\rho_{0}(h)-\rho_{0}(-h)$.

We proceed by describing the mixing by a turbulent diffusivity $\kappa_{T} \sim u_{T} l_{T}$. The size of the largest turbulent eddies in the flow will scale with the height of the mixing region $l_{T} \sim h$ and the turbulent velocity scale can be related to the forcing in the flow by $u_{T} \sim u$ that we obtain from (5.1), giving

$$
\kappa_{T} \sim\left(\frac{\Delta \rho g h}{\bar{\rho}}\right)^{\frac{1}{2}} h .
$$

In scaling the size of turbulent fluctuations $l_{T} \sim h$, we are ignoring any global overturning of the flow and any non-equilibrium acceleration.

In the two-layer classical case, the size of the mixing region is easily defined as the region that has changed density from the initial conditions. The only way a region of fluid in the upper layer can change density is if it contains fluid that began in the lower layer. This is not true for the stratified case as there are parcels of fluid of the complete range of densities in the tank in the initial conditions for both the upper and lower layers. Therefore it is useful to consider a one-dimensional passive scalar $\phi$ (such as a dye), which marks fluid originating from the upper layer (initially $\phi=1$ for $z \leqslant 0$ and $\phi=0$ for $z<0)$.

Assuming the profile evolves self-similarly, we introduce a similarity variable $\xi=\frac{z}{h(t)}$, where $\phi(z, t)=\phi(\xi)$, rescaling the flow by the instantaneous height of the mixed region. Although the stratification introduces an additional length-scale which will always be present, there is some experimental evidence for the stratified case exhibiting features of self-similarity in the dye profiles of Lawrie \& Dalziel (2011a).

The rate of increase of total amount of passive scalar in the lower half of the domain will be equal to the flux of the scalar though $z=0$. Assuming this is controlled by turbulent diffusion then

$$
\frac{\partial}{\partial t} \int_{-\infty}^{0} \phi \mathrm{d} z=\left.\kappa_{T} \frac{d \phi}{d z}\right|_{z=0} .
$$

Introducing our similarity variable we find

$$
\frac{d h}{d t} \int_{-\infty}^{0} \phi(\xi) \mathrm{d} \xi=\left.\frac{\kappa_{T}}{h} \frac{d \phi}{d \xi}\right|_{z=0} .
$$

We can simplify this considerably, under self similarity, as $\int_{-\infty}^{0} \phi(\xi) \mathrm{d} \xi$ and $\left.\frac{d \phi}{d \xi}\right|_{z=0}$ are constant with time, yielding the dimensionally obvious relation $h \dot{h} \sim \kappa_{T}$. Combining this 
with (5.2) we obtain the relation

$$
\frac{d h}{d t} \sim \sqrt{\frac{\Delta \rho g h}{\bar{\rho}}} .
$$

As $\Delta \rho$ is the density difference across $h$, we can define an instantaneous Atwood number $A(h)=\frac{\Delta \rho(h)}{2 \bar{\rho}}$, i.e. the Atwood number across the mixing region at a instant in time for a stratified system,

$$
A(h)=\frac{\rho_{0}(h)-\rho_{0}(-h)}{\rho_{0}(h)+\rho_{0}(-h)},
$$

where $\rho_{0}(z)=\rho(z, t=0)$. This can be written in terms of the equations that are used to describe the initial density profiles in $\S 3$,

$$
A(\hat{h})=A_{0} 2 f(\hat{h}),
$$

where $\hat{h}=\frac{h}{z_{n}}$. Substituting $A=\frac{\Delta \rho}{2 \bar{\rho}}$ into (5.5), we obtain

$$
\frac{d h}{d t} \sim \sqrt{A g h}
$$

We can compare this expression to the classical case, where $A$ is constant throughout the instability $\left(A(h)=A_{0}\right)$ and $h=\alpha A_{0} g t^{2}$, to write this equation in terms of the constant $\alpha$

$$
\frac{d h}{d t}=2 \sqrt{\alpha A g h}
$$

A related differential equation (with constant $A$ ) was obtained for the classical case by Ristorcelli \& Clark (2004) who showed it can be derived directly from the Navier-Stokes equations, using an assumption of self-similarity.

For the case of a linear density profile, (5.9) yields the analytical solution

$$
h=\frac{z_{n}}{2} \sin ^{2}\left(t \sqrt{\frac{2 \alpha A_{0} g}{z_{n}}}\right) .
$$

Unfortunately, for the two quadratic profiles the analytical solution is not so compact and must be expressed in terms of hyper-geometric functions. The model predicts the final height of the mixing region to be $\frac{h_{\infty}}{z_{n}}=\frac{1}{\sqrt{2}}$ for the quadratic with increasing density gradient case $\left(\frac{d^{2} \rho}{d z^{2}}>0\right) ; \frac{h_{\infty}}{z_{n}}=\frac{1}{2}$ for the linear case $\left(\frac{d^{2} \rho}{d z^{2}}=0\right)$; and $\frac{h_{\infty}}{z_{n}}=1-\frac{1}{\sqrt{2}}$ for the quadratic with decreasing density gradient case $\left(\frac{d^{2} \rho}{d z^{2}}<0\right)$. This is the same as the final height of the mixing region for the perfect mixing profiles. A plot of the predictions of the model against non-dimensional time is shown in figure 12a. Each of these curves have the same $z_{n}$ and hence different $h_{\infty}$. The insert shows how the evolution compares if $z_{n}$ for each profile is rescaled such that $h_{\infty}$ for all of the profiles is $\frac{1}{2}$.

The linear case is symmetric around $\frac{z_{n}}{4}$ : the growth of the mixing region accelerates until this point and decelerates thereafter. For the quadratic with increasing density gradient, the turning point in $\dot{h}$ occurs after the half way point, $h_{\infty} / 2$, and for the quadratic with decreasing density gradient the turning point occurs before.

The approach used for deriving this model is different from those used in Lawrie \& Dalziel (2011a), who assumed a functional form for the density profile within the mixing region. However, the final model is similar to that derived in the present paper. The model in Lawrie \& Dalziel (2011a), which was derived only for the linear case, can be 


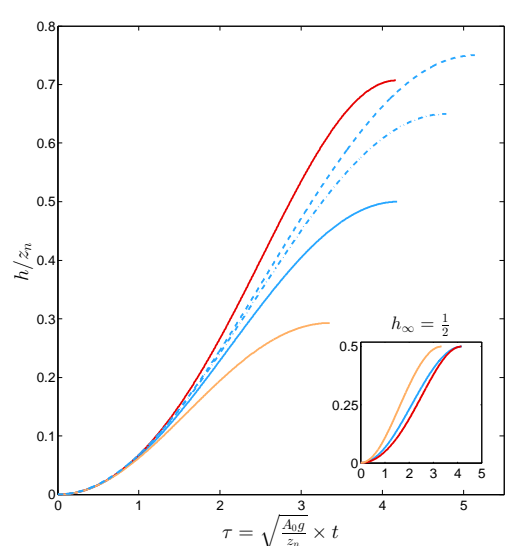

(a)

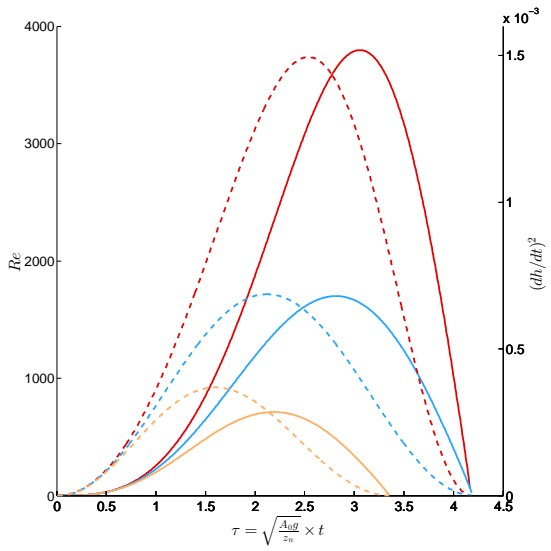

(b)

Figure 12: Model prediction for (a) height of the mixing region predicted by the turbulent diffusion model (solid line) and the model derived in Lawrie \& Dalziel (2011a) (dashed $\chi=\frac{3}{4}$, dash-dot $\chi=0.65$, where $\chi$ is a fitting constant in the model defined in 5.11), inset shows the model predictions with density profiles adjusted such that $h_{\infty}=1 / 2$; (b) Reynolds number (solid line) and $(d h / d t)^{2}$ which is proportional to kinetic energy density (dashed line) with time for $A_{0}=A(t=0)=0.01$. The dark grey line (red on-line) shows the quadratic with increasing density gradient, mid-grey line (blue on-line) shows the linear profile and the light grey line (orange on-line) shows the quadratic with decreasing density gradient.

written as

$$
\frac{\mathrm{d} h}{\mathrm{~d} t} \sim \sqrt{g h A_{0}\left(1-\frac{h}{\chi z_{n}}\right)} .
$$

where $\chi \leqslant \frac{3}{4}$ and must be fitted to experiments. This model is plotted on figure $12 \mathrm{a}$ (using the same constant of proportionality as we used for (5.9)), with two values of the constant $\chi$, the maximum value (dashed line) and that found from fitting to experiments in Lawrie \& Dalziel (2011a) (dash-dot line). As $\chi$ must be fitted to experiments and the constant of proportionality is not clear in (5.11), this model does not predict $h_{\infty}$. If $\chi$ were set at the maximum $\frac{3}{4}$ then the mixing region would extend to $\pm \frac{3 z_{n}}{4}$ and would have a mixing efficiency of $\eta=1$.

The model derived in the present paper, (5.9), essentially predicts a value of $\chi=\frac{1}{2}$ and allows for a direct comparison between a stratified model for $h$ and previous models for the classical instability (by casting the constant of proportionality in (5.8) in terms of the classically derived $\alpha$ ). This new model is also valid in general for any symmetric stratification confined Rayleigh-Taylor instability, unlike (5.11) which is only valid for the linear profile case.

We shall compare the model predictions with our experimental observations shortly. First, however, we shall explore a number of features of the model. Using our model we can express the Reynolds number, $R e=\frac{h \dot{h}}{\nu}$, in terms of the instantaneous Atwood number,

$$
R e=\frac{\sqrt{\alpha A g h^{3}}}{\nu}
$$


Thus the maximum Reynolds number in the flow scales as $R e \sim \sqrt{A_{0}}$, but the instantaneous values will vary as some function of $h$ during the development of the flow. Figure $12 \mathrm{~b}$ shows the Reynolds number (solid lines) and the kinetic energy density $\left(\left(\frac{d h}{d t}\right)^{2}\right.$; dashed lines), against time for each of the three cases, with an initial Atwood number of 0.01 (which is at the lower end of the range for the experiments shown in figure 11) and $z_{n}=0.2 \mathrm{~m}$. In each case, the peak Reynolds number occurs after the peak velocity in the flow, as although the velocity has started to decay (due to the effect of the stable stratification), the length-scale of eddies in the mixing region is still increasing. This was also seen in the model derived by Lawrie \& Dalziel (2011a) for the linear case. After this peak the model predicts a sharp decline in the Reynolds number as the stable stratification slows the growth of the mixing region. In reality, the Reynolds number of the flow does not reach zero when the mixing region reaches its equilibrium height as there is still kinetic energy remaining in the mixing region (as seen in figure 6c), a feature not captured in the model.

The assumption of an instantaneous balance between buoyancy and inertia is equivalent to asserting the flow evolves at a constant (negative) Richardson number within the mixing region. Specifically we can define a Richardson number within the mixing region as

$$
R i=-2 g A \frac{h}{(\mathrm{~d} h / \mathrm{d} t)^{2}} .
$$

This is different to the gradient Richardson number, $R i_{e}$, for the edge of the mixing region we defined earlier. Substituting for $\frac{\mathrm{d} h}{\mathrm{~d} t}$ in (5.13) we find that the model predicts a $R i$ which is constant and negative

$$
R i=-\frac{1}{2 \alpha}
$$

This is also the case for the model for the classical two layer instability $\left(h=\alpha A_{0} g t^{2}\right)$. In experiments, kinetic energy remains after all negative density gradients in the system have been removed, therefore the Richardson number within the mixing region in reality increases to become positive in the late stages of the instability. Mixing continues until $R i$ is larger than some critical $R i_{c}$.

The model derived in Lawrie \& Dalziel (2011a) does not make the assumption of an instantaneous balance between buoyancy and inertia. There $R i_{\chi}$ was defined as

$$
R i_{\chi} \sim \frac{2 \hat{h}-1}{1-\hat{h} / \chi} .
$$

Again we can see that if $\chi=1 / 2$ then we recover the constant, negative Richardson number found above. Increasing $\chi$ allows mixing to continue after the profile is overall stable by allowing a greater proportion of $E_{a}$ to be released into $E_{k}$. This results in a positive $R i$ at late times.

\subsection{Comparison with experiments}

A comparison of the predictions of this model with experiments using dyed fluid is shown in figure 13. Dyed fluid attenuates light that passes through it, as described by Allgayer \& Hunt (2012), Cenedese \& Dalziel (1998) and Holford \& Dalziel (1996), and used in the investigation of Rayleigh-Taylor instability by Dalziel et al. (2008). In the present work, attenuation was used to determine how the height of the mixed region evolves in time. The upper layer was dyed with a constant concentration of methylene blue dye. Figure 13 shows the evolution of the horizontally-averaged dye concentration. The spatial variation present due to the finite speed of the barrier was removed by taking each column of a 
video and shifting it according to the time at which the barrier passed that point and then averaging. The colour in figure 13 shows $\left|\phi-\phi_{0}\right|$, where $\phi$ is the dye concentration (normalized by the initial concentration in the upper layer, $0>\phi>1$ ) and $\phi_{0}=\phi(t=0)$.

In making this comparison between experiment and model, we use our measurements of $\rho_{0}(z)$ for each experiment to determine $A(h)$. The effect of the barrier on the initial conditions for the flow is included in the model by initialising $h$ with the thickness of the barrier $(2.55 \mathrm{~mm})$, effectively setting the scale of the initial perturbations. The only fitting constant in this model is $\alpha$.

In the past there has been much disagreement over the value of the constant $\alpha$ between experiments and simulations of classical Rayleigh-Taylor. Many authors now believe that this is due to differences in initial conditions (e.g. Dalziel et al. (1999), Ramaprabhu \& Andrews (2004), Tsiklashvili et al. (2012)), though another suggested explanation for the variation in $\alpha$ is the difference in Schmidt number $\left(S c=\frac{\nu}{D}\right.$, where $\nu$ is the kinematic viscosity and $D$ is the mass diffusivity) between experiments and numerics (Glimm et al. 2001). In experimental procedures there is inevitably some memory of initial conditions, as large wavelength perturbations of the order of the dimensions of the container will be present (Youngs 1984; Dalziel 1993; Snider \& Andrews 1994). Experiments of two-layer Rayleigh-Taylor instability with widely varying experimental procedures have found a value of $\alpha$ that is around 0.07 (Dalziel 1993; Ramaprabhu \& Andrews 2004; Tsiklashvili et al. 2012), though other experimental studies have found values that are somewhat lower, e.g. 0.057 (Dimonte et al. 2004). In comparing our model to experiments we have simply taken $\alpha=0.07$. We have not attempted to determine $\alpha$ from our present experiments, but instead aim to show that the value found in experiments of the classical instability is still appropriate in our model for the stratified case.

The model appears to work well in the cases shown in figures 13(b) and (c), and less well in the case shown in (a) (the quadratic with increasing density gradient). Even in (a), the initial behaviour of the mixing region (up to $\sim 3 \mathrm{~s}$ ) is well modelled. Although the model does not appear to predict the exact height with time of the mixing region well in all cases, it does correctly predict the time constant for the development of the mixing region.

When the initial density profile is a quadratic with increasing density gradient, there is initially no density gradient immediately to either side of the unstable interface. This means that this density profile is more likely to be strongly affected by the initial conditions than the other two profiles, as there are no strong density gradients near the unstable interface to damp out disturbances and flatten the vortical structures produced by withdrawal of the barrier. This could be the reason for the discrepancy for $t>3 \mathrm{~s}$ between model and experiment in figure 13a. The model assumes that the length-scale controlling the development of the mixing region scales with the height of the mixing region. It is possible that the length scale of the initial perturbation remains important and acts as a barrier to the growth of the turbulent length scale. This would result in the slower growth that we see in experiments of this case.

One of the limitations of the model derived in the present paper, (5.9), is that it does not allow for sorting within the central region as sorting indicates that there is not an instantaneous balance between buoyancy and inertia. The model cannot predict any transport of turbulent kinetic energy in the flow, which removes the possibility of modelling mixing of the stable profiles above and below the central turbulent region. The lack of any mechanism for sorting within the central mixing region is the reason why the model slightly under predicts the height of the mixing region at late times. A possible next step would be a model that includes some transport of kinetic energy, releasing the assumption of an instantaneous balance. 


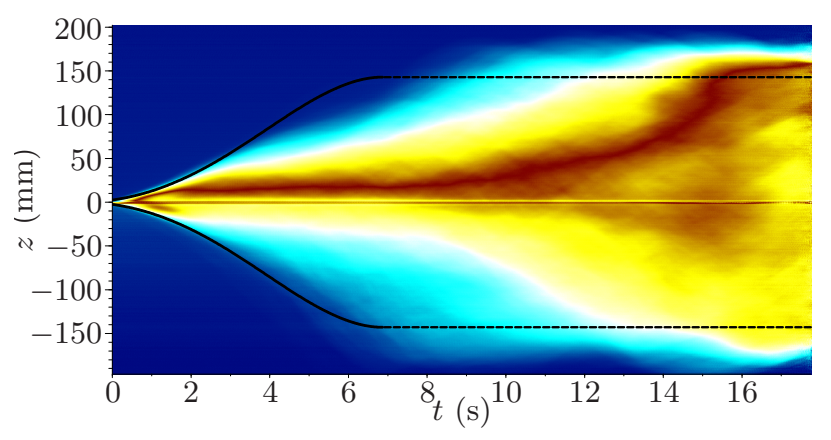

(a)

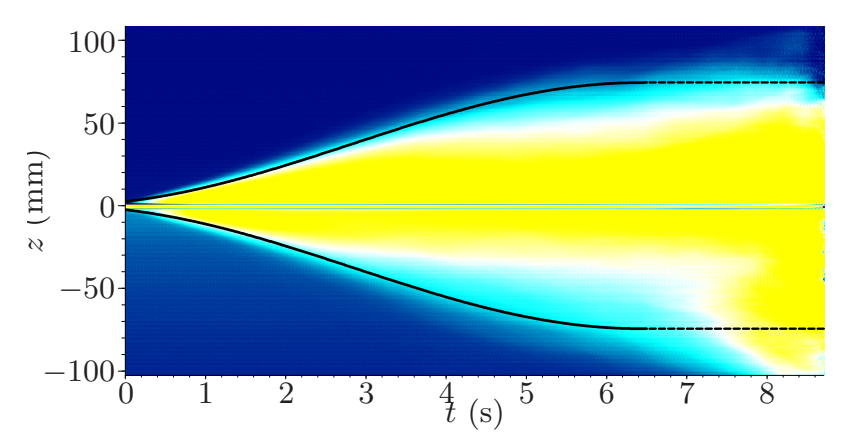

(b)

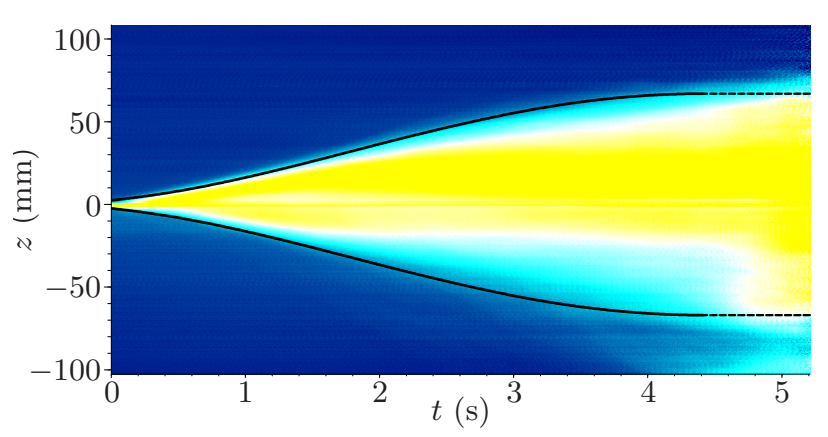

(c)

Figure 13: Comparison with dyed experiments with the model $\dot{h}=2 \sqrt{\alpha A g h}$, where $\alpha=0.07$ and $A(h)$ is calculated from the measured initial density profile. $h(t=0)$ is equal to the thickness of the barrier. Initial density profile: (a) quadratic with increasing density gradient, (b) linear, (c) quadratic with decreasing density gradient. The colour shows $\left|\phi-\phi_{0}\right|$, where $\phi$ is the dye concentration normalized by the initial dye concentration in the upper layer. Dashed line on (a) is an adjusted model with the size of turbulent eddies scaling as $l_{T} \sim \lambda_{b}$. 


\section{Conclusions}

We have shown that the final state of Rayleigh-Taylor instability between two stablystratified layers has an almost perfectly mixed central region, surrounded by relatively unchanged stable stratifications. The mixing efficiency is generally greater than $95 \%$ of the perfect mixing efficiency $\eta_{\mathrm{p}}$, which is calculated assuming the final state has a central well mixed region, surrounded by regions which are unaltered from the initial state. The mixing efficiency increases as the curvature of the density field $\frac{\mathrm{d}^{2} \rho}{\mathrm{d}|z|^{2}}$ decreases, though the height mixed, as a proportion of the height of the stratification, decreases with decreasing $\frac{\mathrm{d}^{2} \rho}{\mathrm{d}|z|^{2}}$.

In some cases there is evidence of a small amount of mixing in the stable stratification above and below the central mixing region. This occurs when the gradient Richardson number at the edge of the mixing region $\left(R i_{e}\right)$ is small when the mixing region reaches a height at which the profile is overall stable (the perfect mixing height). This relationship between Richardson number and mixing of a stable interface by turbulence generated in a Rayleigh-Taylor unstable region was also found by Jacobs \& Dalziel (2005).

Some sorting within the central region appears in the final density profiles of all experiments. Sorting within the central region decreases the mixing efficiency (as compared to the perfect mixing efficiency) as stability is achieved by sorting within the gravitationally unstable region rather than by mixing. In general, for Atwood numbers $A<0.04$, sorting within the central region had a stronger effect than mixing of the stable stratifications outside the central region, resulting in a mixing efficiency slightly below the perfect mixing value.

If the central region becomes homogenised (making the profile overall stable) only mixing of the stable profiles above and below the central region can increase the potential energy (as no further mixing is possible in a region of constant density). This occurred when the Atwood number was high $(A>0.04)$, but as the kinetic energy must first be converted into available potential energy (by moving a parcel of fluid into a region where it is surrounded by parcels of a different density), mixing by this mechanism is much less efficient compared to mixing which is directly driven by gravitational instability. Therefore it seems unlikely that the mixing efficiency will ever increase much beyond the perfect mixing value.

Surprisingly, the amount of mixing decreases with the introduction of additional kinetic energy from the removal of the barrier. When the barrier speed was increased, increasing the kinetic energy present in the initial conditions, less mixing and more sorting was apparent in the central region of the final density profile (resulting in a stronger residual stratification), leading to a decrease in the mixing efficiency even if the additional energy input is not taken into account. (Had the final stratification remained unchanged, then the kinetic energy input from the barrier would have represented a decrease in the actual mixing efficiency.)

A theoretical model was developed for the height of the mixing region, $\dot{h}=2 \sqrt{\alpha A g h}$, where $A=A(h)$ is the instantaneous Atwood number across the mixing region, $\alpha=$ 0.07 (a typical experimental value in classical RTI) and $g$ is gravitational acceleration. The only fitting constant in this model is $\alpha$, a value for which was taken from earlier two-homogeneous layer Rayleigh-Taylor instability experiments. This model was seen to match the growth of the mixing region well for early times in experiments and also matches the overall time-scale for the growth of the mixing region. The departure from this model in the case when there is no density gradient close to the unstable interface may be due to the effect of the initial perturbations imposed by the barrier.

We have shown that the maximum mixing efficiency possible is greater than 0.75 . The 
assumption that is sometimes made, that $\eta$ can be approximated by 0.2 in all mixing situations is unjustified. In regions of the ocean that have unstable density gradients (e.g. river outflows and regions where surface cooling makes the mixed layer of the ocean convectively unstable) there is the potential for much higher mixing efficiencies. It is clear that mixing efficiency can vary widely, particularly where unstable density gradients exist in an otherwise stably stratified flow.

We would like to thank Professor David Youngs and Dr Robin Williams for many useful discussions. This work was funded by EPSRC (grant number EP/P505445/1) and an AWE CASE award (AWE contract number 30174006). This work is (c) British Crown Copyright 2014/AWE.

\section{REFERENCES}

Allgayer, D. M. \& Hunt, G. R. 2012 On the application of the light-attenuation technique as a tool for non-intrusive buoyancy measurements. Experimental Thermal and Fluid Science 38, 257-261.

Alon, U., Hecht, J., Ofer, D. \& Shvarts, D. 1995 Power Laws and Similarity of RayleighTaylor and Richtmyer-Meshkov Mixing Fronts at All Density Ratios.

Andrews, M. J. \& DAlziel, S. B. 2010 Small Atwood number Rayleigh-Taylor experiments. Philosophical transactions. Series A, Mathematical, physical, and engineering sciences 368 (1916), 1663-79.

Boffetta, G., Mazzino, A., Musacchio, S. \& Vozella, L. 2010 Rayleigh-Taylor instability in a viscoelastic binary fluid. Journal of Fluid Mechanics 643, 127.

Савот, W. H. \& Cоок, A. W. 2006 Reynolds number effects on Rayleigh-Taylor instability with possible implications for type Ia supernovae. Nature Physics 2 (8), 562-568.

Cenedese, C. \& Dalziel, S. B. 1998 Concentration and depth fields determined by the light transmitted though a dyed solution. In Measurement.

Cook, A. W., Савот, W. H. \& Miller, P. L. 2004 The mixing transition in Rayleigh-Taylor instability. Journal of Fluid Mechanics 511, 333-362.

DAlziel, S. B. 1993 Rayleigh-Taylor instability: experiments with image analysis. Dynamics of Atmospheres and Oceans 20 (1-2), 127-153.

Dalziel, S. B. 1994 Molecular Mixing in Rayleigh-Taylor Instability. Tech. Rep. September.

Dalziel, S. B., Linden, P. F. \& Youngs, D. L. 1999 Self-similarity and internal structure of turbulence induced by Rayleigh-Taylor instability. Journal of Fluid Mechanics 399, 1-48.

Dalziel, S. B., Patterson, Michael D., Caulfield, C. P. \& Coomaraswamy, imran a. 2008 Mixing efficiency in high-aspect-ratio RayleighTaylor experiments. Physics of Fluids 20 (6), 065106.

Dimonte, G., Youngs, D. L., Dimits, A., Weber, S., Marinak, M., Wunsch, S., Garasi, C., Robinson, A., Andrews, M. J., Ramaprabhu, P., Calder, A. C., Fryxell, B., Biello, J., Dursi, L., MacNeice, P., Olson, K., Ricker, P., Rosner, R., Timmes, F., Tufo, H., Young, Y.-N. \& Zingale, M. 2004 A comparative study of the turbulent Rayleigh-Taylor instability using high-resolution three-dimensional numerical simulations: The Alpha-Group collaboration. Physics of Fluids 16 (5), 1668.

Glimm, James, Grove, J. W., Li, X. L., Oh, W. \& Sharp, D. H. 2001 A Critical Analysis of Rayleigh-Taylor Growth Rates. Journal of Computational Physics 169 (2), 652-677.

Grinstein, Fernando F., Margolin, Len G. \& Rider, William J. 2007 Implicit large eddy simulation: computing turbulent fluid dynamics.

Holford, J. M. \& DAlziel, S. B. 1996 Measurements of layer depth during baroclinic instability in a two-layer flow. Applied Scientific Research 56 (2-3), 191-207.

Inogamov, N. $\sim$ A., Oparin, A. $\sim$ M., Dem'Yanov, A. $\sim$ Y., Dembitskit, L. $\sim$ N. \& Khokhlov, V. A. 2001 On Stochastic Mixing Caused by the Rayleigh Taylor Instability. Soviet Journal of Experimental and Theoretical Physics 92, 715-743.

Jacobs, J. W. \& DAlziEL, S. B. 2005 RayleighTaylor instability in complex stratifications. Journal of Fluid Mechanics 542 (-1), 251. 
Lawrie, A G. W. \& Dalziel, S. B. $2011 a$ Rayleigh-Taylor mixing in an otherwise stable stratification. Journal of Fluid Mechanics 688, 507-527.

Lawrie, A G. W. \& Dalziel, S. B. $2011 b$ Turbulent diffusion in tall tubes. II. Confinement by stratification. Physics of Fluids 23 (8), 085110.

Linden, P. F., Redondo, J. M. \& Youngs, D. L. 1994 Molecular mixing in Rayleigh-Taylor instability. Journal of Fluid Mechanics 265, 97-124.

Lister, J. R., Kerr, R. C., Russell, N. J. \& Crosby, A. 2011 Rayleigh-Taylor instability of an inclined buoyant viscous cylinder. Journal of Fluid Mechanics 671, 313-338.

Lorenz, E. N. 1954 Available Potential Energy and the Maintenance of the General Circulation. Tellus 7 (2), 157-167.

Mueschke, N. J., Schilling, O., Youngs, D. L. \& Andrews, M. J. 2009 Measurements of molecular mixing in a high-Schmidt-number Rayleigh-Taylor mixing layer. Journal of Fluid Mechanics 632, 17.

Munk, W. \& Wunsch, C. 1998 Abyssal recipes II: energetics of tidal and wind mixing. Deep Sea Research Part I: Oceanographic Research Papers 45 (12), 1977-2010.

Peltier, W. R. \& Caulfield, C. P. 2003 Mixing Efficiency in Stratified Shear Flows. Annual Review of Fluid Mechanics 35 (1), 135-167.

Petrasso, Richard D. 1994 Rayleigh's Challenge Endures. Nature Physics .

Prastowo, Tupto, Griffiths, Ross W., Hughes, Graham O. \& Hogg, Andrew Mc C. 2008 Mixing efficiency in controlled exchange flows. Journal of Fluid Mechanics 600, 235244 .

Ramaprabhu, P. \& Andrews, M. J. 2004 Experimental investigation of Rayleigh-Taylor mixing at small Atwood numbers. Journal of Fluid Mechanics 502, 233-271.

RAYLEIGH 1900 Investigation of the character of the equilibrium of an incompressible heavy fluid of variable density. Scientific Papers 2, 598.

READ, K.I. 1984 Experimental investigation of turbulent mixing by Rayleigh-Taylor instability. Physica D: Nonlinear Phenomena 12 (1-3), 45-58.

RistorCelli, J. R. \& ClARK, T. T. 2004 Rayleigh-Taylor turbulence: self-similar analysis and direct numerical simulations. Journal of Fluid Mechanics 507, 213-253.

Sharp, D. H. 1984 An overview of Rayleigh-Taylor Instability. Physica D: Nonlinear Phenomena 12D, 3-18.

Snider, D. M. \& Andrews, M. J. 1994 RayleighTaylor and shear driven mixing with an unstable thermal stratification. Physics of Fluids 6 (10), 3324.

TAIlleux, R. 2009 Understanding mixing efficiency in the oceans: do the nonlinearities of the equation of state for seawater matter? Ocean Science Discussions 6 (1), 371-387.

TAllleux, R. 2013 Available Potential Energy and Exergy in Stratified Fluids. Annual Review of Fluid Mechanics 45 (1), 35-58.

TAYLOR, G. 1950 The Instability of Liquid Surfaces when Accelerated in a Direction Perpendicular to their Planes. I. Proceedings of the Royal Society A: Mathematical, Physical and Engineering Sciences 201 (1065), 192-196.

Tsiklashvili, V., Colio, P. E. R., Likhachev, O. \& Jacobs, J. W. 2012 An experimental study of small Atwood number Rayleigh-Taylor instability using the magnetic levitation of paramagnetic fluids. Physics of Fluids 24 (5), 052106.

Turner, J. S. 1973 Buoyancy effects in fluids.

Winters, K. B., Lombard, P. N., Riley, J. J. \& D'Asaro, E. A. 1995 Available potential energy and mixing in density-stratified fluids. Journal of Fluid Mechanics 289 (-1), 115128.

Wunsch, C. \& Ferrari, R. 2004 Vertical Mixing, Energy, and the General Circulation of the Oceans. Annual Review of Fluid Mechanics 36 (1), 281-314.

Youngs, D. L. 1984 Numerical simulation of turbulent mixing by Rayleigh-Taylor instability. Physica D: Nonlinear Phenomena 12 (1-3), 32-44. 